SUPPORTING INFORMATION for

\title{
SUB-NANOMETER STRUCTURE OF AN ASYMMETRIC MODEL MEMBRANE: INTERLEAFLET COUPLING INFLUENCES DOMAIN PROPERTIES
}

Frederick A. Heberle, Drew Marquardt, Milka Doktorova, Barbara Geier, Robert F. Standaert, Peter Heftberger, Benjamin Kollmitzer, Jonathan D. Nickels, Robert A. Dick, Gerald W. Feigenson, John Katsaras, Erwin London and Georg Pabst 
Table S1 Bilayer components and neutron scattering length density calculations. Molecular volumes of deuterated components are assumed to be identical to their protiated counterpart.

\begin{tabular}{|c|c|c|c|c|}
\hline Component & Formula & $\mathbf{V}\left[\AA^{3}\right]$ & $b[\mathbf{f m}]^{a}$ & $\operatorname{SLD}\left[\mathrm{fm} \AA^{-3}\right]^{b}$ \\
\hline 16:0-18:1 chains & $\mathrm{C}_{32} \mathrm{H}_{64}$ & $915.0^{\mathrm{c}}$ & -26.6 & -0.029 \\
\hline $16: 0(\mathrm{~d} 31)-18: 1$ chains & $\mathrm{C}_{32} \mathrm{H}_{33} \mathrm{D}_{31}$ & $915.0^{\mathrm{c}}$ & 296.1 & 0.322 \\
\hline di-16:0(d31) chains & $\mathrm{C}_{30} \mathrm{D}_{62}$ & $810.4^{\mathrm{c}}$ & 613.0 & 0.756 \\
\hline $\mathrm{PC}$ head in $\mathrm{D}_{2} \mathrm{O}$ & $\mathrm{C}_{10} \mathrm{H}_{18} \mathrm{NO}_{8} \mathrm{P}$ & $331^{d}$ & 60.1 & 0.181 \\
\hline $\mathrm{PC}(\mathrm{d} 13)$ head in $\mathrm{D}_{2} \mathrm{O}$ & $\mathrm{C}_{10} \mathrm{H}_{5} \mathrm{D}_{13} \mathrm{NO}_{8} \mathrm{P}$ & $331^{d}$ & 195.4 & 0.590 \\
\hline Heavy water & $\mathrm{D}_{2} \mathrm{O}$ & 30.0 & 19.2 & 0.636 \\
\hline
\end{tabular}

${ }^{\mathrm{a}}$ coherent neutron scattering length; ${ }^{\mathrm{b}}$ scattering length density, $\mathrm{b} / \mathrm{V} ;{ }^{\mathrm{c}}$ ref 1 ; ${ }^{\mathrm{d}} \mathrm{ref} 2$ 
Table S2 Leaflet compositions for an asymmetric LUV sample prepared from POPC acceptor and POPC- $d H C$ donor, determined from GC, ${ }^{1} \mathrm{H}-\mathrm{NMR}$, and SANS modeling. Data columns from left to right indicate: bilayer mole fraction; component fraction in the outer versus inner leaflet; mole fraction of all lipids found in the outer leaflet; inner leaflet mole fraction; and outer leaflet mole fraction.

\begin{tabular}{|l|c|c|c|c|c|}
\hline \multicolumn{1}{|c|}{ Component } & $\chi$ & $\boldsymbol{f}^{\text {out }}$ & $\mathbf{X}^{\text {out }}$ & $\chi^{\text {in }}$ & $\chi^{\text {out }}$ \\
\hline POPC (acceptor) & $0.64^{\mathrm{a}}$ & $0.29^{\mathrm{b}}$ & \multirow{2}{*}{$0.494^{\mathrm{c}}$} & $0.90^{\mathrm{d}}\left(0.45^{\mathrm{e}}\right)$ & $0.38^{\mathrm{d}}\left(0.19^{\mathrm{e}}\right)$ \\
\cline { 1 - 4 } POPC- $d H C$ (donor) & $0.36^{\mathrm{a}}$ & & & $0.10^{\mathrm{d}}\left(0.05^{\mathrm{e}}\right)$ & $0.62^{\mathrm{d}}\left(0.31^{\mathrm{e}}\right)$ \\
\hline Total & 1.00 & & & $1.00^{\mathrm{d}}\left(0.50^{\mathrm{e}}\right)$ & $1.00^{\mathrm{d}}\left(0.50^{\mathrm{e}}\right)$ \\
\hline
\end{tabular}

${ }^{\mathrm{a}}$ from GC; ${ }^{\mathrm{b}}$ from ${ }^{1} \mathrm{H}-\mathrm{NMR} ;{ }^{\mathrm{c}}$ from SANS structural modeling; ${ }^{\mathrm{d}}$ leaflet mole fraction; ${ }^{\mathrm{e}}$ total bilayer mole fraction

Table S3 Leaflet compositions for an asymmetric LUV sample prepared from POPC- $d H C$ acceptor and POPC donor, determined from GC, ${ }^{1} \mathrm{H}-\mathrm{NMR}$, and SANS modeling. Data columns as described in Table S2 legend.

\begin{tabular}{|l|c|c|c|c|c|}
\hline \multicolumn{1}{|c|}{ Component } & $\chi$ & $\boldsymbol{f}^{\text {out }}$ & $\mathbf{X}^{\text {out }}$ & $\chi^{\text {in }}$ & $\chi^{\text {out }}$ \\
\hline POPC- $d H C$ (acceptor) & $0.62^{\mathrm{a}}$ & & \multirow{3}{*}{$0.50^{\mathrm{c}}$} & $0.84^{\mathrm{d}}\left(0.42^{\mathrm{e}}\right)$ & $0.40^{\mathrm{d}}\left(0.20^{\mathrm{e}}\right)$ \\
\cline { 1 - 3 } \cline { 5 - 6 } & & & $0.16^{\mathrm{d}}\left(0.08^{\mathrm{e}}\right)$ & $0.60^{\mathrm{d}}\left(0.30^{\mathrm{e}}\right)$ \\
\hline POPC (donor) & 1.00 & & & $1.00^{\mathrm{d}}\left(0.50^{\mathrm{e}}\right)$ & $1.00^{\mathrm{d}}\left(0.50^{\mathrm{e}}\right)$ \\
\hline
\end{tabular}

${ }^{\mathrm{a}}$ from GC; ${ }^{b}$ from ${ }^{1} \mathrm{H}-\mathrm{NMR} ;{ }^{\mathrm{c}}$ from SANS structural modeling; ${ }^{\mathrm{d}}$ leaflet mole fraction; ${ }^{\mathrm{e}}$ total bilayer mole fraction

Table S4 Leaflet compositions for an asymmetric LUV sample prepared from POPC- $d H$ acceptor and DPPC- $d C$ donor, determined from GC, ${ }^{1} \mathrm{H}-\mathrm{NMR}$, and SANS modeling. Data columns as described in Table S2 legend.

\begin{tabular}{|l|c|c|c|c|c|}
\hline \multicolumn{1}{|c|}{ Component } & $\chi$ & $\boldsymbol{f}^{\text {out }}$ & $\mathbf{X}^{\text {out }}$ & $\chi^{\text {in }}$ & $\chi^{\text {out }}$ \\
\hline POPC- $d H$ (acceptor) & $0.82^{\mathrm{a}}$ & & \multirow{2}{*}{$0.512^{\mathrm{c}}$} & $0.98^{\mathrm{d}}\left(0.48^{\mathrm{e}}\right)$ & $0.66^{\mathrm{d}}\left(0.34^{\mathrm{e}}\right)$ \\
\cline { 1 - 4 } DPPC- $d C$ (donor) & $0.18^{\mathrm{a}}$ & $0.95^{\mathrm{b}}$ & & $0.02^{\mathrm{d}}\left(0.01^{\mathrm{e}}\right)$ & $0.34^{\mathrm{d}}\left(0.18^{\mathrm{e}}\right)$ \\
\hline Total & 1.00 & & & $1.00^{\mathrm{d}}\left(0.49^{\mathrm{e}}\right)$ & $1.00^{\mathrm{d}}\left(0.52^{\mathrm{e}}\right)$ \\
\hline
\end{tabular}

${ }^{\mathrm{a}}$ from GC; ${ }^{\mathrm{b}}$ from ${ }^{\mathrm{l} H-N M R} ;{ }^{\mathrm{c}}$ from SANS structural modeling; ${ }^{\mathrm{d}}$ leaflet mole fraction; ${ }^{\mathrm{e}}$ total bilayer mole fraction 
Table S5 Refinement of symmetric POPC vesicles. Best fit parameters for differently contrasted symmetric POPC bilayers. Data were both individually and jointly modeled with a symmetric four slab scattering length density profile as described in Supporting Information Section S8. Model parameters from top to bottom indicate: area per lipid $\left(A_{L}\right)$; number of headgroup waters $\left(n_{W}\right)$; headgroup thickness $\left(D_{H}\right)$; and hydrocarbon thickness $\left(D_{C}\right)$.

\begin{tabular}{|c|c|c|c|c|c|}
\hline \multirow{2}{*}{ Param } & \multicolumn{4}{|c|}{ Individual refinement } & \multirow{2}{*}{$\begin{array}{c}\text { Joint } \\
\text { refinement }\end{array}$} \\
\cline { 2 - 6 } & POPC & POPC- $d H$ & POPC- $d C$ & POPC-dHC & inner/outer \\
\cline { 2 - 6 } & inner/outer & inner/outer & inner/outer & inner/outer & 67.5 \\
\hline$A_{L}\left[\AA^{2}\right]^{\mathrm{a}}$ & 68.6 & 68.1 & 66.7 & 63.5 & 7.2 \\
\hline$n_{W}^{\mathrm{a}}$ & 8.2 & 2.0 & 8.0 & 2.0 & 8.0 \\
\hline$D_{H}[\AA]^{\mathrm{b}}$ & 8.4 & 5.7 & 8.0 & 6.1 & 13.5 \\
\hline$D_{C}[\AA]^{\mathrm{b}}$ & 13.4 & 13.4 & 13.7 & 14.4 & \\
\hline
\end{tabular}

${ }^{\mathrm{a}}$ variable parameter; ${ }^{\mathrm{b}}$ derived from other model parameters, parameter uncertainties are estimated to be $<2 \%$

Table S6 Refinement of asymmetric POPC vesicles. Best fit parameters for two differently contrasted asymmetric POPC bilayers. Data were both individually and jointly modeled with an asymmetric four slab scattering length density profile as described in Supporting Information Section S8. Model parameters as in the Table S5 legend.

\begin{tabular}{|c|c|c|c|c|c|c|}
\hline \multirow{3}{*}{ Param } & \multicolumn{4}{|c|}{ Individual refinement } & \multirow{2}{*}{\multicolumn{2}{|c|}{$\begin{array}{c}\text { Joint } \\
\text { refinement }\end{array}$}} \\
\hline & \multicolumn{2}{|c|}{ POPC acc./POPC-dHC don. } & \multicolumn{2}{|c|}{ POPC- $d H C$ acc./POPC don. } & & \\
\hline & inner & outer & inner & outer & inner & outer \\
\hline$A_{L}\left[\AA^{2}\right]^{\mathrm{a}}$ & 64.8 & 66.4 & 65.9 & 65.9 & 64.8 & 66.4 \\
\hline$n_{W}{ }^{\mathrm{a}}$ & 2 & 2 & 3.6 & 1.6 & 2 & 2 \\
\hline$D_{H}[\AA]^{\mathrm{b}}$ & 6.0 & 5.9 & 5.7 & 6.7 & 6.0 & 5.9 \\
\hline$D_{C}[\AA]^{\mathrm{b}}$ & 14.1 & 13.8 & 13.9 & 13.9 & 14.1 & 13.8 \\
\hline
\end{tabular}

${ }^{\mathrm{a}}$ variable parameter; ${ }^{\mathrm{b}}$ derived from other model parameters, parameter uncertainties are estimated to be $<2 \%$ 
Table S7 Refinement of chemically asymmetric aLUVs. Bilayer structural best-fit parameters for an aLUV sample prepared from POPC- $d H$ acceptor and DPPC- $d C$ donor, determined from fitting SANS data to an asymmetric two phase model. Model parameters from top to bottom indicate: mole fraction of DPPC within each leaflet $\left(\chi_{D P P C}\right)$; area per lipid $\left(A_{L}\right)$; number of headgroup waters $\left(n_{W}\right)$; headgroup thickness $\left(D_{H}\right)$; hydrocarbon thickness $\left(D_{C}\right)$; and phase area fraction $\left(\alpha_{k}\right)$.

\begin{tabular}{|c|c|c|c|c|}
\hline \multirow{2}{*}{ Parameter } & \multicolumn{2}{|c|}{ Phase 1 } & \multicolumn{2}{c|}{ Phase 2 } \\
\cline { 2 - 5 } & inner & outer & inner & outer \\
\hline$\chi_{D P P C}$ & $0.019^{\mathrm{a}}$ & $0.024^{\mathrm{c}}$ & $0.019^{\mathrm{b}}$ & $0.82^{\mathrm{c}}$ \\
\hline$A_{L}\left[\AA^{2}\right]$ & $64.0^{\mathrm{d}}$ & $64.0^{\mathrm{d}}$ & $64.0^{\mathrm{d}}$ & $56.7^{\mathrm{c}}$ \\
\hline$n_{W}^{\mathrm{a}}$ & 7 & 7 & 7 & 7 \\
\hline$D_{H}[\AA]^{\mathrm{e}}$ & 8.5 & 8.5 & 8.5 & 9.6 \\
\hline$D_{C}[\AA]^{\mathrm{e}}$ & 14.3 & 14.3 & 14.3 & 14.6 \\
\hline$\alpha_{k}{ }^{\mathrm{b}}$ & \multicolumn{2}{|c}{0.63} & & 0.37 \\
\hline
\end{tabular}

${ }^{a}$ fixed parameter; ${ }^{b}$ constrained from matter balance; ${ }^{c}$ variable parameter; ${ }^{d}$ jointly variable parameters; ${ }^{\mathrm{e}}$ derived from other model parameters, parameter uncertainties are estimated to be $<2 \%$

Table S8 LUV size and polydispersity determined by dynamic light scattering before and after centrifugal filtration. Uncertainty is estimated to be $\pm 5 \mathrm{~nm}$. All measurements were performed at room temperature.

\begin{tabular}{|c|c|c|c|c|}
\hline \multirow{2}{*}{ Sample } & \multicolumn{2}{|c|}{ Before centrifugal filtration } & \multicolumn{2}{c|}{ After centrifugal filtration $^{\mathbf{b}}$} \\
\cline { 2 - 5 } & Diameter [nm] & Rel. polydisp. & Diameter [nm] & Rel. polydisp. \\
\hline POPC LUV & 130.2 & 0.27 & 128.4 & 0.25 \\
\hline POPC LUV & 123.3 & 0.23 & 128.9 & 0.36 \\
\hline POPC- $d H$ LUV $^{\mathrm{a}}$ & 125.1 & 0.23 & 131.1 & 0.28 \\
\hline POPC- $d C$ LUV $^{\mathrm{a}}$ & 116.2 & 0.22 & 118.9 & 0.28 \\
\hline DPPC- $d C$ LUV $^{\mathrm{a}}$ & 116.6 & 0.12 & 116.3 & 0.24 \\
\hline $\begin{array}{c}\text { (POPC- } d H) \text { in/(DPPC- } \\
d C) \text { out aLUV }\end{array}$ & 131.2 & 0.24 & 129.6 & 0.30 \\
\hline
\end{tabular}

${ }^{a}$ vesicles prepared in $20 \mathrm{mM} \mathrm{NaCl}$; ${ }^{b} 2-4$ concentration/dilution cycles were performed as described in Supporting Information section S1. ${ }^{c}$ Measurement before centrifugual filtration is of symmetric extruded acceptor LUVs, measurement after centrifugal filtration is of asymmetric LUVs. 


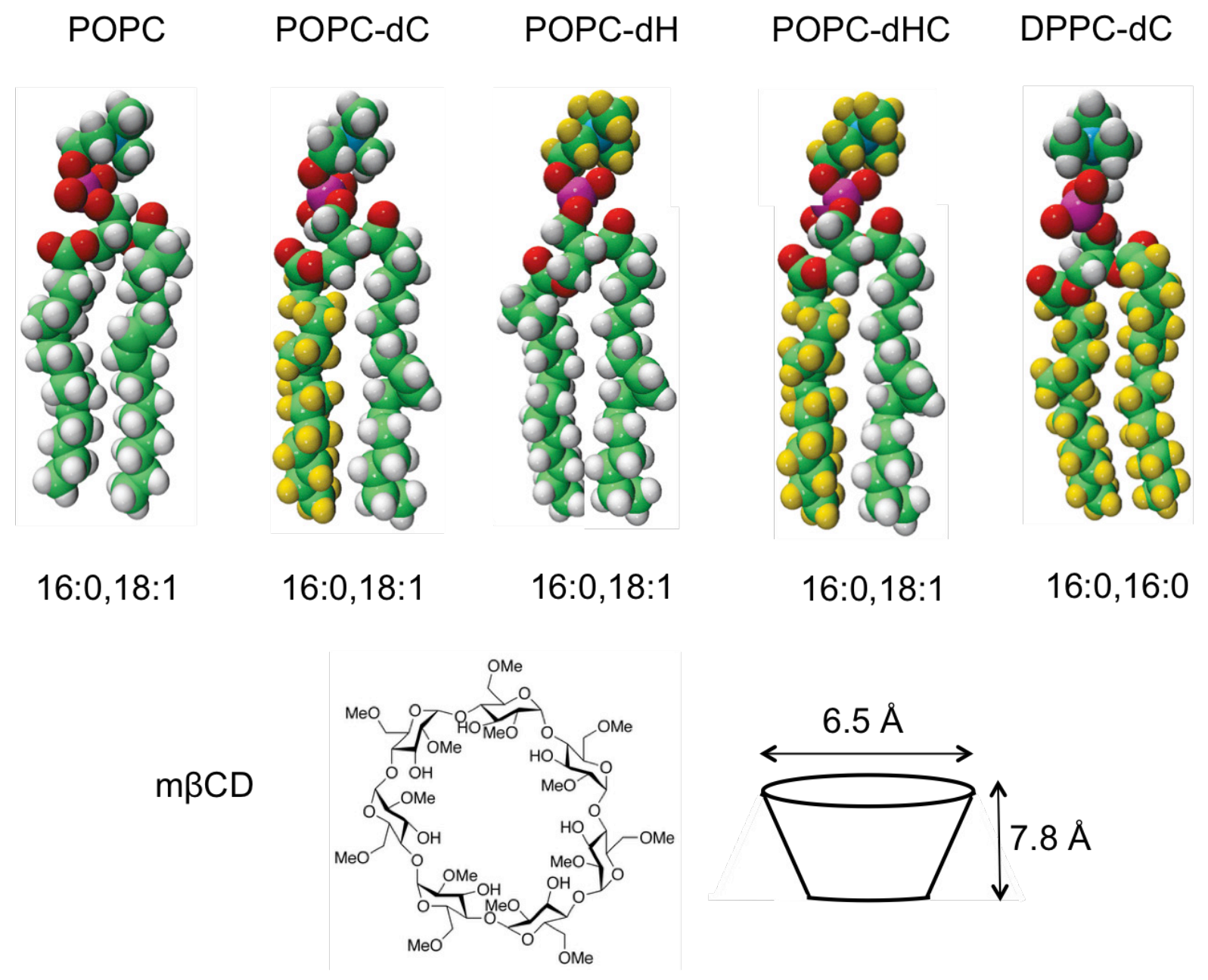

Figure S1 | Chemical structures of phospholipids and methyl-beta-cyclodextrin $(\mathrm{m} \beta \mathrm{CD})$ used in this study. Lipids are displayed in space fill representation with white indicating hydrogen and yellow indicating deuterium. The label underneath each lipid shows the number of carbons and the number of double bonds in the $s n-1$ and $s n-2$ chains, respectively. 


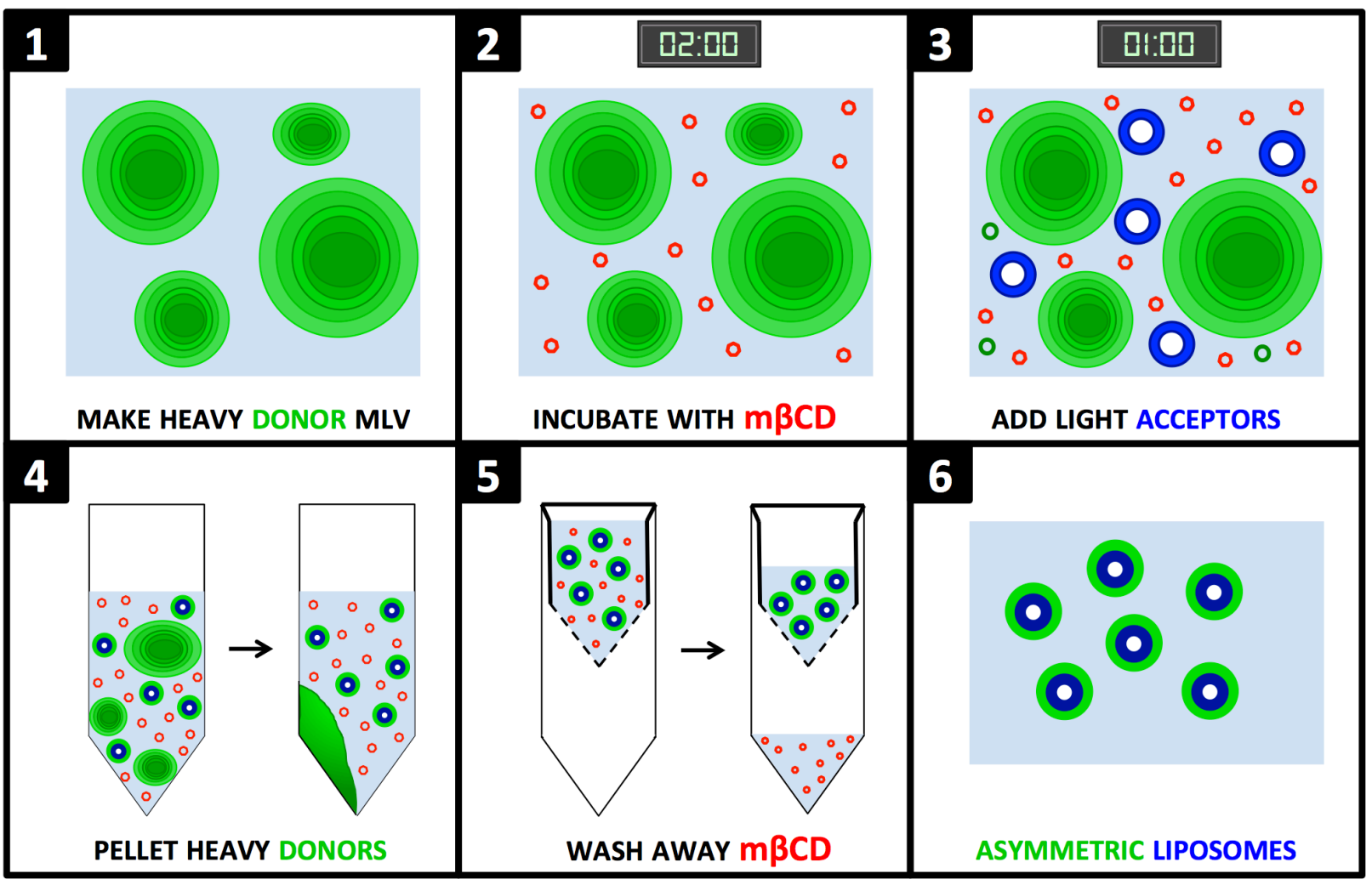

Figure S2 | Protocol for the preparation of sucrose-free asymmetric liposomes. Step 1, donor multilamellar vesicles (MLVs) are prepared in 20\% (w/w) sucrose, then diluted 20-fold with water and centrifuged (supernatant is discarded). Step 2, aqueous methyl-beta cyclodextrin $(\mathrm{m} \beta \mathrm{CD}, 35 \mathrm{mM})$ is added to the MLV pellet in an 8:1 $\mathrm{m} \beta \mathrm{CD}$ :donor ratio and incubated for $2 \mathrm{~h}$ at room temperature while stirring. Step 3, a suspension of acceptor large unilamellar vesicles (LUVs) is added to the donor/m $\beta C D$ sample to achieve a desired donor:acceptor molar ratio and an $\mathrm{m} \beta \mathrm{CD}$ concentration of $\sim 29 \mathrm{mM}$, then incubated for $1 \mathrm{~h}$ at room temperature while stirring. Acceptor LUVs can be prepared in low osmolarity buffer (e.g., $10-30 \mathrm{mM} \mathrm{NaCl}$ ) to balance residual solutes remaining after the asymmetric sample preparation. Depending on the $\mathrm{m} \beta \mathrm{CD}$ concentration, a small fraction of the heavy donor vesicles may be dissolved and reformed as light small unilamellar vesicles (SUVs). Step 4, the mixture is diluted 8-fold with $\mathrm{H}_{2} \mathrm{O}$, then centrifuged for $30 \mathrm{~min}$ at $20 \mathrm{~K} \times \mathrm{g}$ (pellet is discarded). Step 5, supernatant from Step 4 is first concentrated to $0.5-1 \mathrm{~mL}$ with a $100 \mathrm{~K} \mathrm{MWCO}$ centrifugal filter. The remaining $\mathrm{m} \beta \mathrm{CD}$ is then removed by repeated dilution (with $\mathrm{H}_{2} \mathrm{O}$ or $\mathrm{D}_{2} \mathrm{O}$, depending on experimental needs) and concentration steps, with the filtrate discarded between steps, to achieve a desired dilution factor. Step 6, asymmetric sucrose-free LUVs in water are recovered from the retentate following the final wash. 

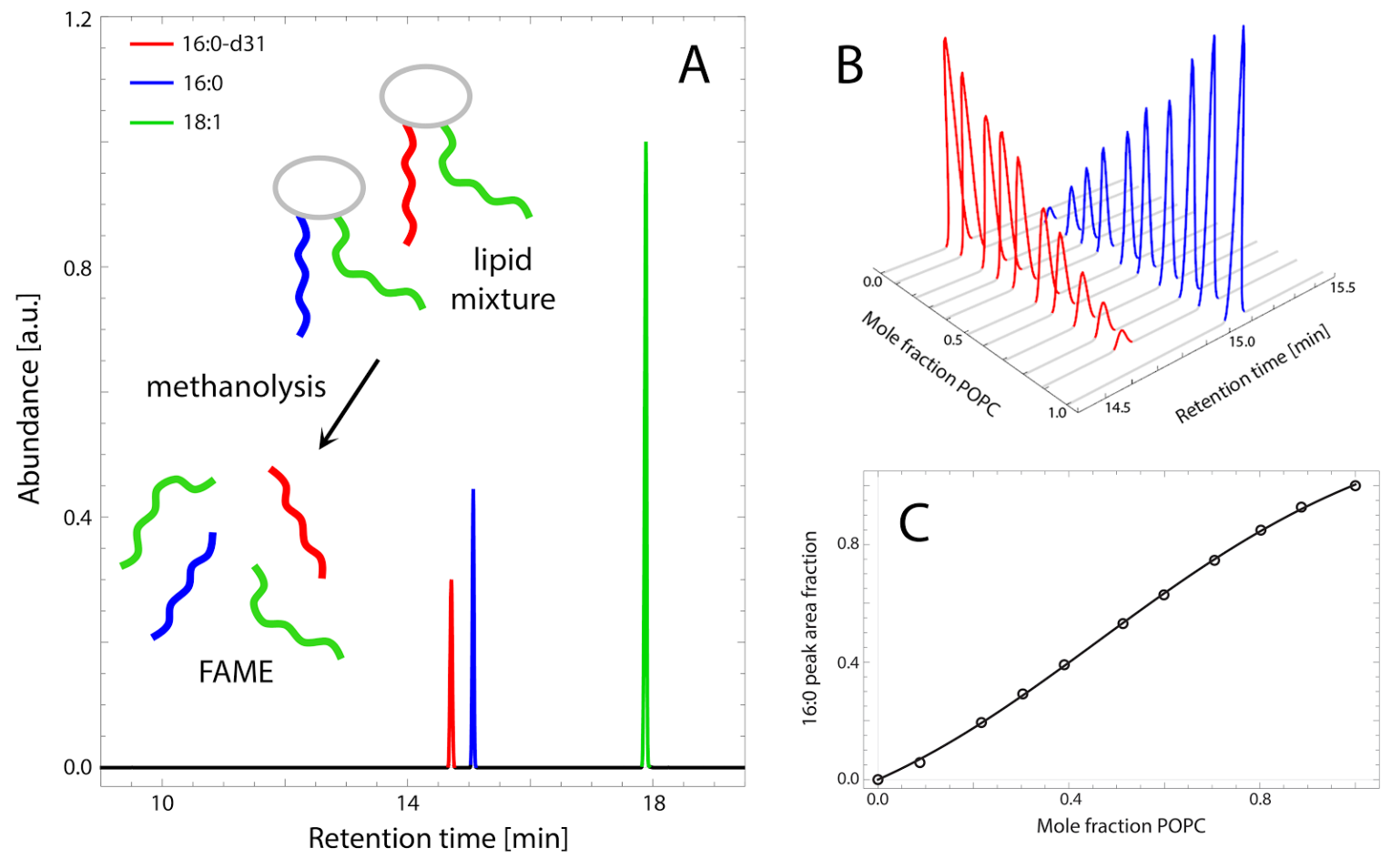

Figure S3 | GC determines the total composition of lipid mixtures. $A$, A lipid sample is subjected to an acid- or base-catalyzed transesterification, converting individual chains to volatile FAMEs suitable for GC analysis (inset cartoon). A binary equimolar mixture of POPC and POPC- $d C$ yields a 2:1:1 ratio of methyl oleate, methyl palmitate, and methyl palmitate-d31, which is reflected in the relative peak areas from the total ion chromatogram. $B$, Changing the mixture composition alters the relative areas of the methyl palmitate and methyl palmitate-d31 peaks. The composition of an unknown sample can therefore be obtained from its peak area fraction. $C$, Detection inefficiencies result in a nonlinear dependence of peak area fraction $v s$. mixture composition. Precise quantitation of an unknown sample requires comparison to a standard curve obtained from mixtures of known composition. 


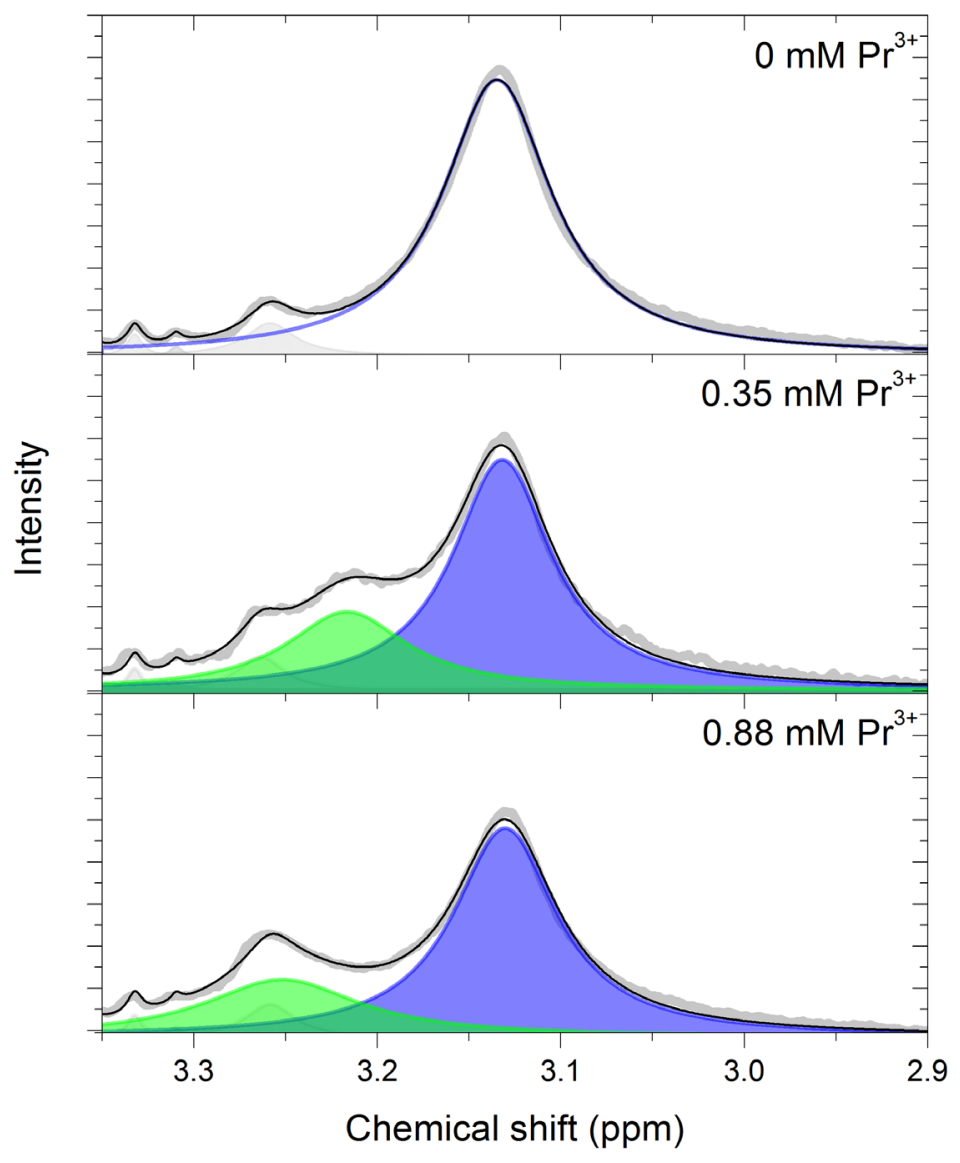

Figure S4 | ${ }^{1} \mathrm{H}-\mathrm{NMR}$ of an aLUV sample prepared from POPC acceptor and POPC-dHC donor. Upper panel, proton NMR shows a single choline resonance from the POPC acceptor in the absence of shift reagent (blue line), in addition to the minor contaminants glycerol and $\mathrm{m} \beta C D$ (gray shading). The sum of all fitted components is shown as a solid black line. Lower panels, addition of $\mathrm{Pr}^{3+}$ selectively shifts outer leaflet choline resonances (green shading), revealing acceptor enrichment in the inner leaflet, where it is inaccessible to the shift reagent (blue shading). 


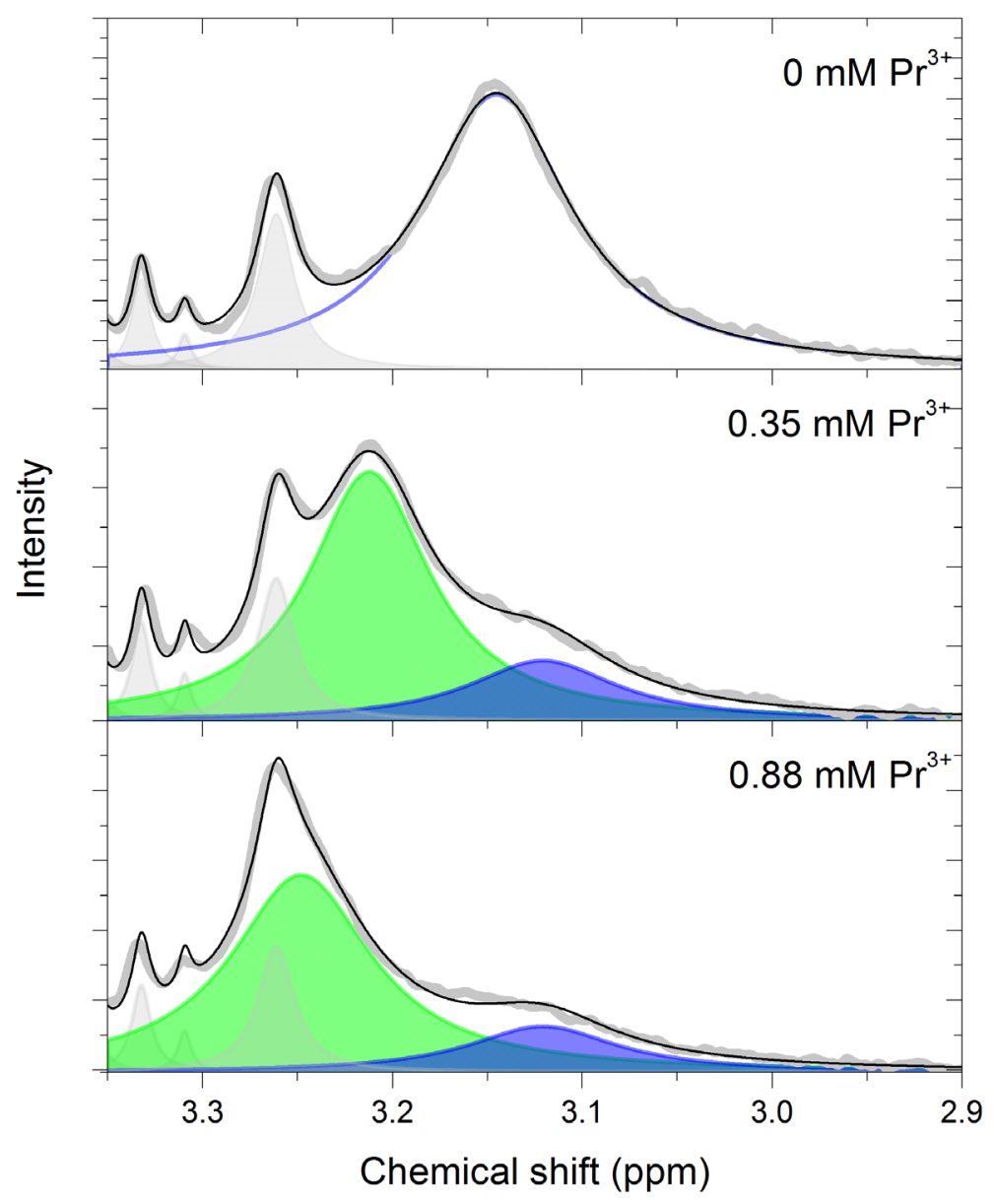

Figure S5 $\mid{ }^{1} \mathrm{H}-\mathrm{NMR}$ of an aLUV sample prepared from POPC-dHC acceptor and POPC donor. Upper panel, proton NMR shows a single choline resonance from POPC donor in the absence of shift reagent (blue line), in addition to the minor contaminants glycerol and $\mathrm{m} \beta \mathrm{CD}$ (gray shading). The sum of all fitted components is shown as a solid black line. Lower panels, addition of $\mathrm{Pr}^{3+}$ selectively shifts outer leaflet choline resonances (green shading), revealing donor enrichment in the outer leaflet. Approximately $20 \mathrm{~mol} \%$ of donor lipid is found in the inner leaflet, where it is inaccessible to the shift reagent (blue shading). 


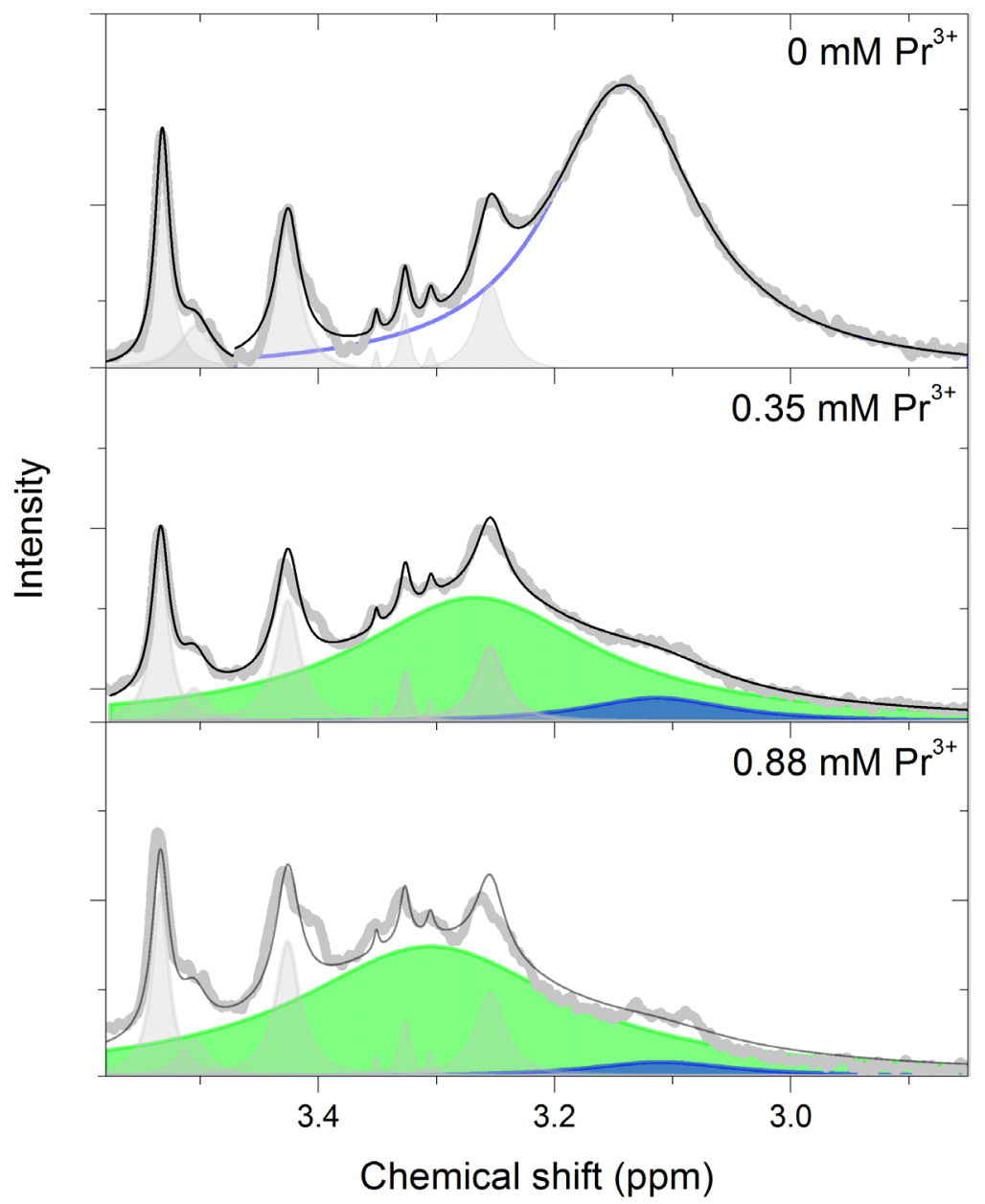

Figure S6 $\mid{ }^{1}$ H-NMR of an asymmetric LUV sample prepared from POPC- $d H$ acceptor and DPPC- $d C$ donor. Upper panel, proton NMR shows a single choline resonance from DPPC- $d C$ donor in the absence of shift reagent (blue line), in addition to the minor contaminants glycerol and $\mathrm{m} \beta \mathrm{CD}$ (gray shading). The sum of all fitted components is shown as a solid black line. Lower panels, addition of $\mathrm{Pr}^{3+}$ selectively shifts outer leaflet choline resonances (green shading), revealing donor enrichment in the outer leaflet. A small fraction $(\sim 6 \mathrm{~mol} \%)$ of donor lipid is found in the inner leaflet, where it is inaccessible to the shift reagent (blue shading). 


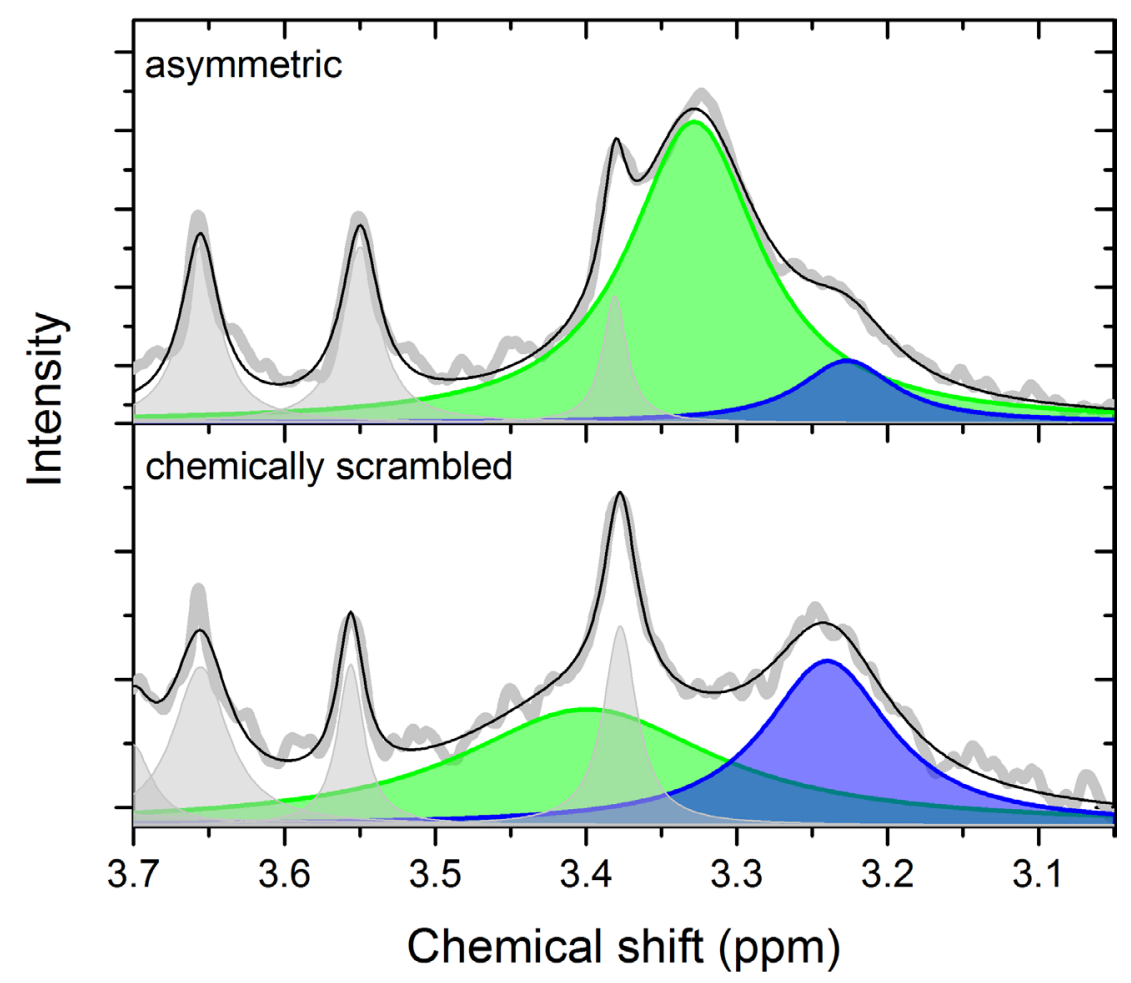

Figure S7 | ${ }^{1} \mathrm{H}-\mathrm{NMR}$ of an aLUV sample prepared from POPC- $d \boldsymbol{H}$ acceptor and POPC donor, before and after chemical scrambling. Upper panel, proton NMR shows the choline resonance from POPC donor in the presence of $\operatorname{Pr}^{3+}$. The large shifted population (green shading) relative to the unshifted population (blue shading) reveals substantial outer leaflet donor enrichment in the aLUV sample. Lower panel, the same sample after chemical scrambling (i.e., removal of water, dissolution in chloroform, and reformation of extruded LUVs) shows shifted and unshifted resonances with nearly equal areas, demonstrating the loss of asymmetry. Minor contaminants glycerol and $\mathrm{m} \beta \mathrm{CD}$ are shown with gray shading. 


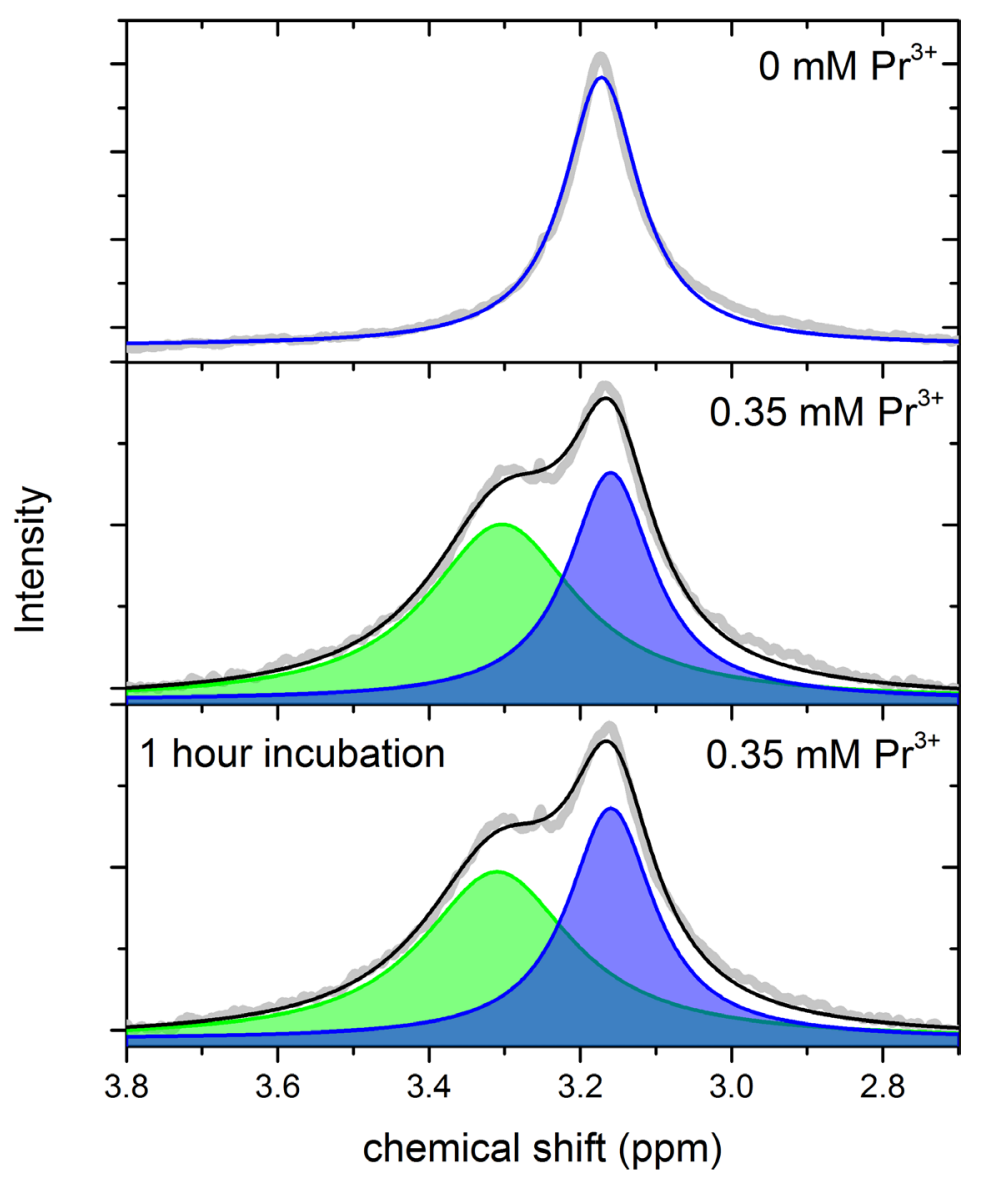

Figure S8 | ${ }^{1}$ H-NMR of a symmetric, phase-separated LUV. A single choline resonance is observed in phase-separated POPC/DPPC (1/1 molar ratio) LUVs in the absence of $\operatorname{Pr}^{3+}$ (upper panel). Distinct resonances of nearly equal area corresponding to inner leaflet (blue shading) and outer leaflet (green shading) choline are observed immediately following external $\operatorname{Pr}^{3+}$ addition (middle panel), and after 1 hour of incubation at room temperature (lower panel), demonstrating that $\operatorname{Pr}^{3+}$ does not penetrate into the vesicle lumen during the $\sim 15$ minute measurement time. 


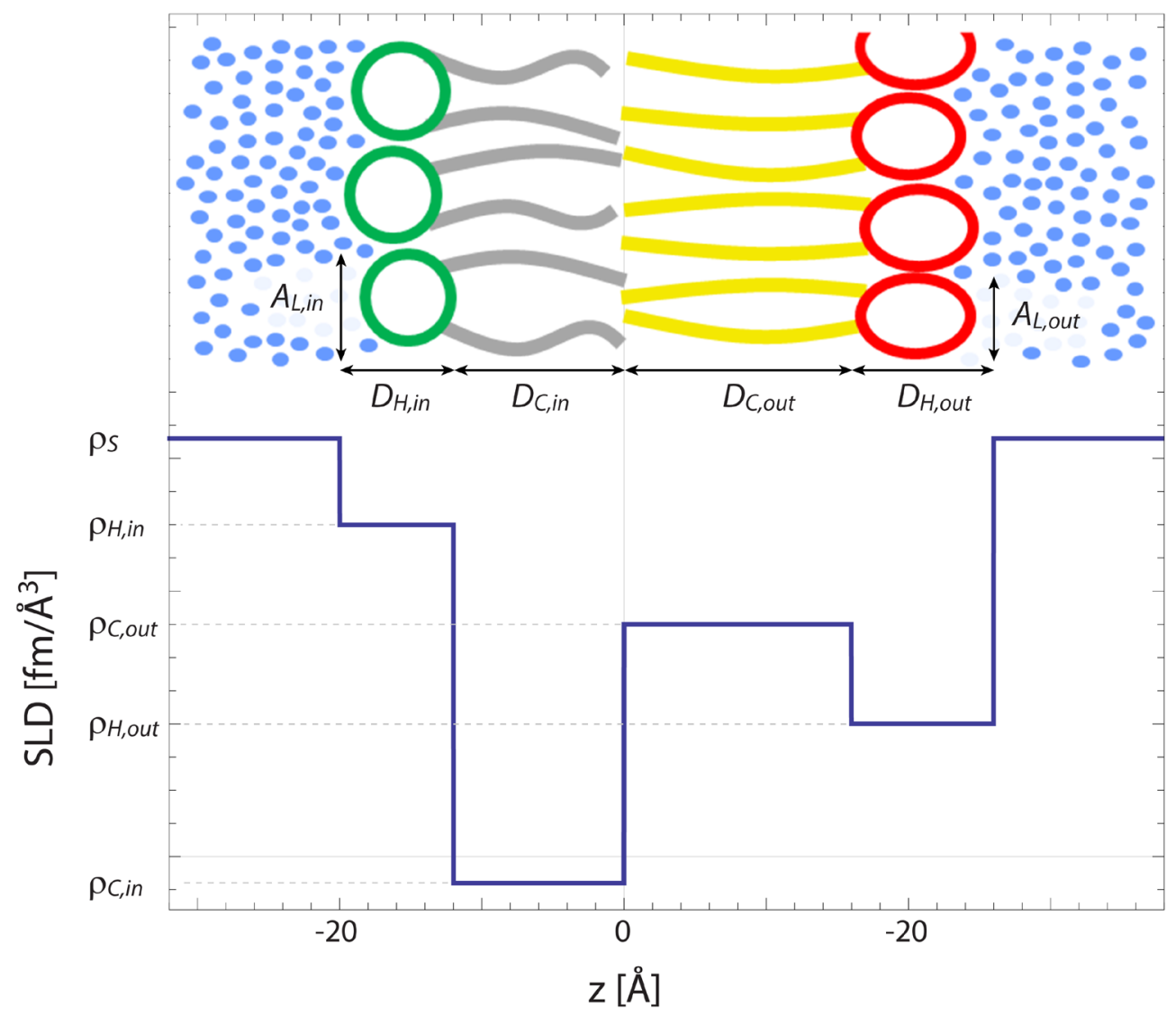

Figure S9 | Four shell scattering length density profile for an asymmetric bilayer. Upper, a schematic representation of an asymmetric bilayer. Lower, the corresponding scattering length density profile is represented by a simplified four slab model with parameters for slab thicknesses and scattering length densities. Parameter symbols are as defined in the Supporting Information text. 


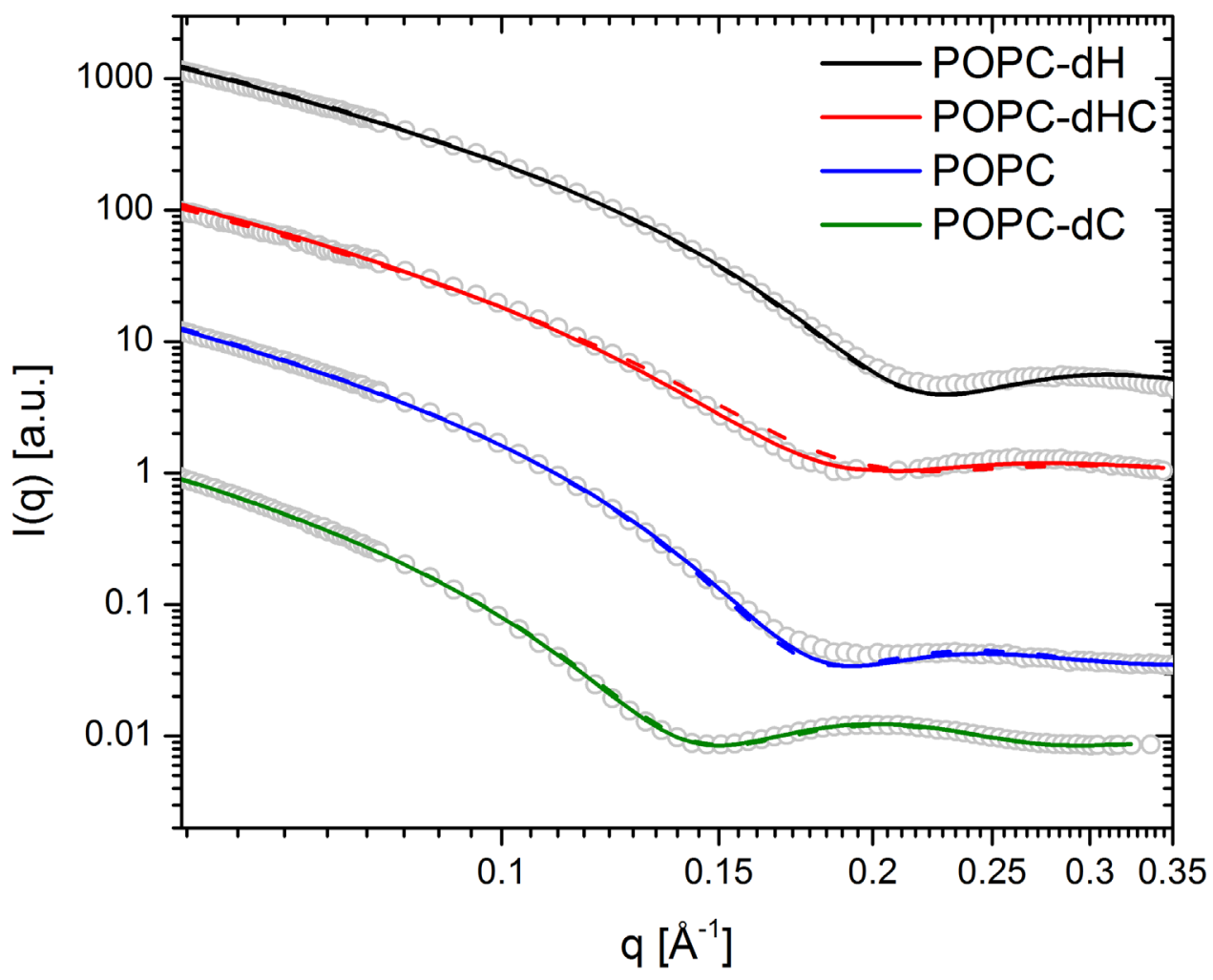

Figure S10 | SANS data and fits for symmetric POPC vesicles. Experimental SANS data (circles) for different contrast symmetric POPC bilayers. Data were modeled individually (solid lines) and jointly (dashed lines) with a symmetric four shell scattering length density profile as described in the Supporting Information text. Predicted scattered intensity is shown as solid lines, with best fit parameters given in Table S5. Data are vertically offset by powers of 10 for clarity. 


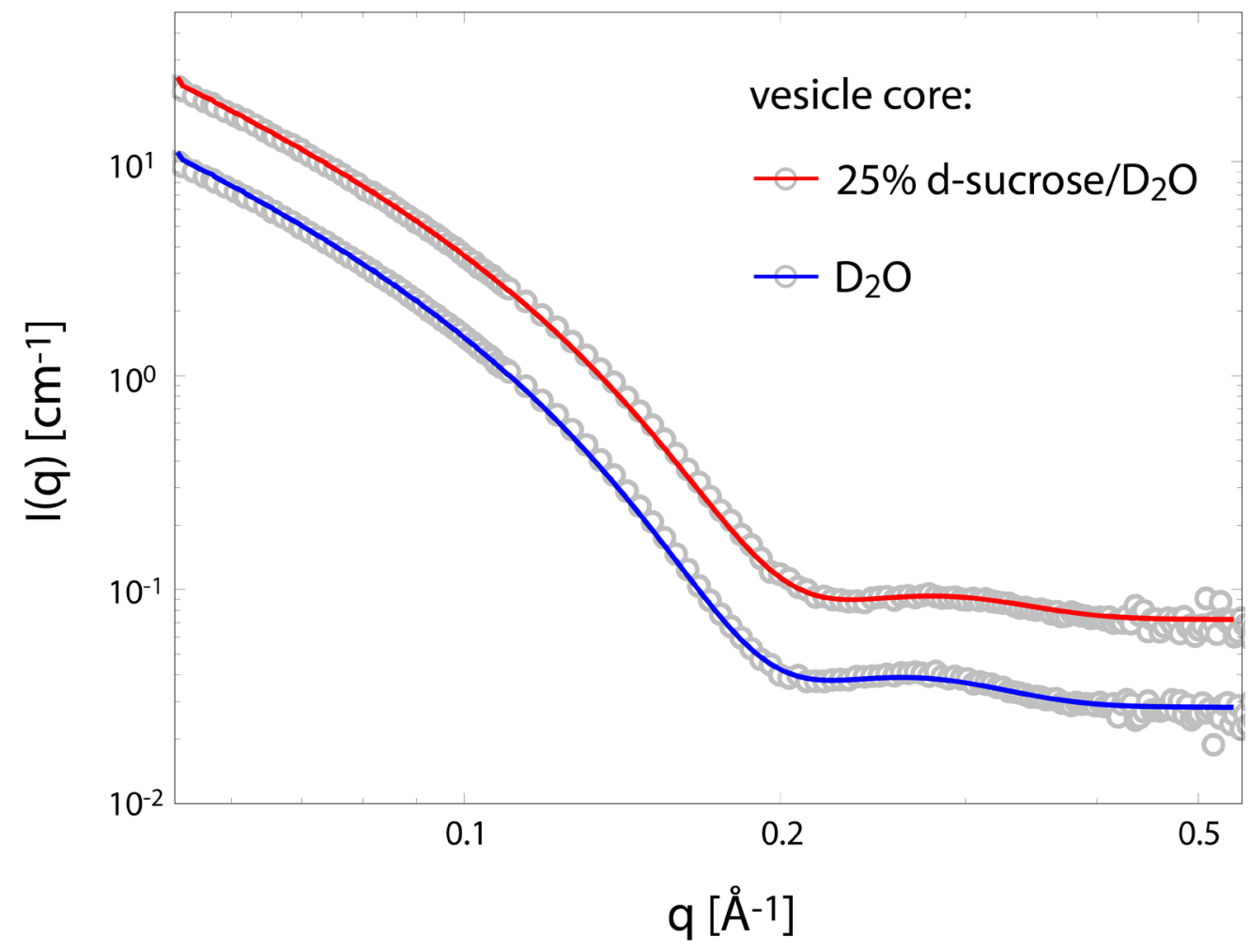

Figure S11 | Osmotic imbalance generates membrane tension and thins POPS vesicles. Experimental SANS data for symmetric $100 \mathrm{~nm}$ diameter POPS LUVs, with different core/solvent conditions. Osmotically stressed POPS vesicles containing a $25 \% \mathrm{~d}$-sucrose core (red) in an external $\mathrm{D}_{2} \mathrm{O}$ solvent show a decreased bilayer thickness $(-1.8 \AA)$ and an increased area per lipid $\left(+4 \AA^{2}\right.$ ) compared to stress-free POPS vesicles prepared in $\mathrm{D}_{2} \mathrm{O}$ (blue), consistent with vesicle swelling and lateral bilayer expansion. Data are vertically offset for clarity. 

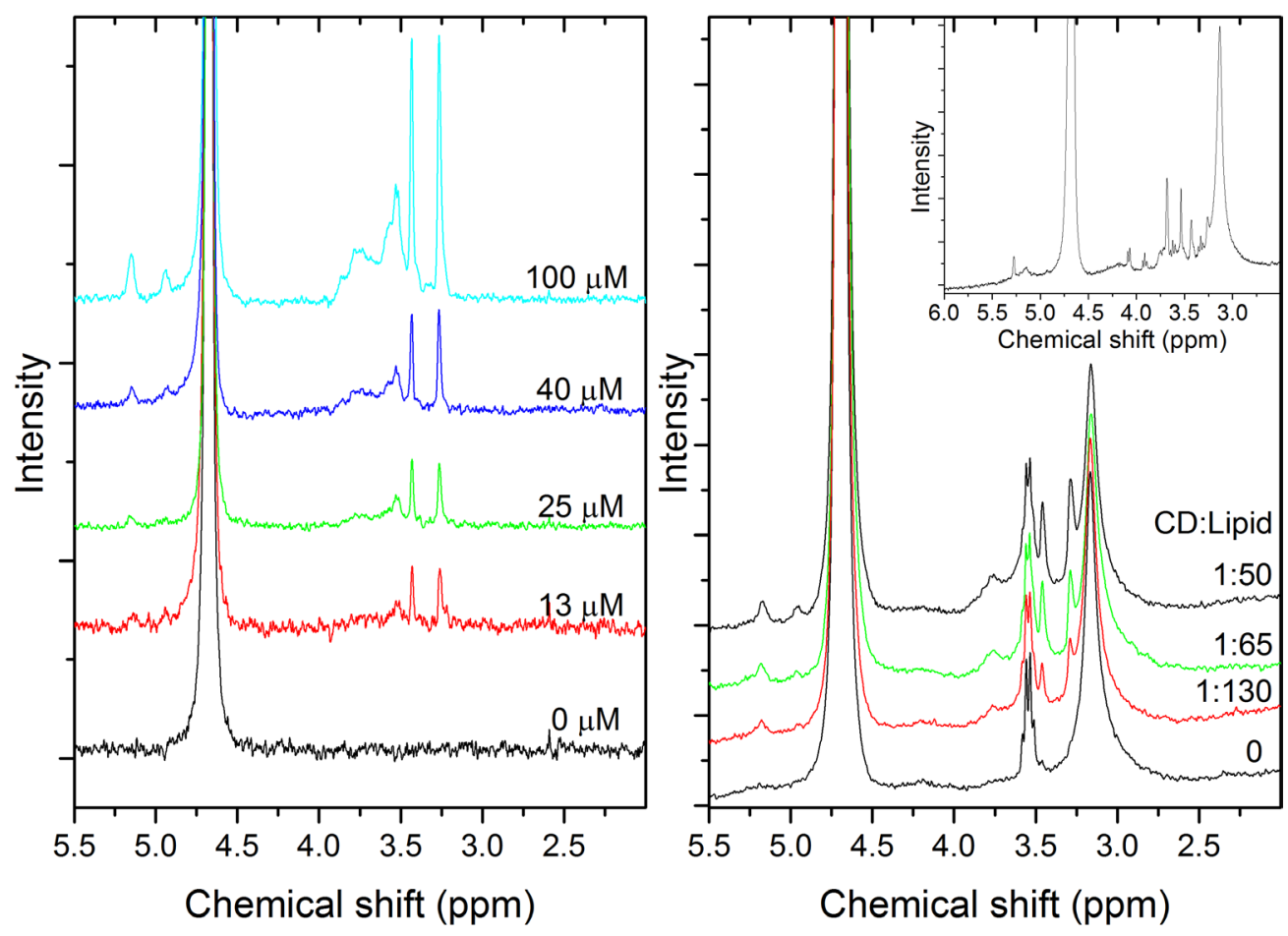

Figure S12 | ${ }^{\mathbf{H}} \mathrm{H}-\mathrm{NMR}$ detection $\mathbf{m} \boldsymbol{\beta C D}$. Titration of $\mathrm{m} \beta \mathrm{CD}$ into $\mathrm{D}_{2} \mathrm{O}$ (left) reveals the solute's detection limit with proton NMR. The characteristic CD peaks occur at $3.29 \mathrm{ppm}$ and $3.46 \mathrm{ppm}$. Comparison of corresponding resonances in an asymmetric LUV sample provides a lower limit for residual $\mathrm{m} \beta C D$ concentration of 1:130 CD:lipid molar ratio. Titration of $\mathrm{m} \beta C D$ into an $\mathrm{LUV} / \mathrm{D}_{2} \mathrm{O}$ dispersion (right) reveals the solute's quantity relative to the lipid (CD:lipid). The choline peak (3.17 ppm) was used to determine CD:lipid ratios. Spectra are vertically offset for clarity. Inset, proton NMR spectra of an asymmetric LUV sample reveals a low quantity of CD relative to lipid ( 7:100 molar ratio). An octet at $3.6 \mathrm{ppm}$ is attributed to trace glycerol contamination originating in the centrifugal filters, despite extensive pre-washing as described in the Supporting Information Section S2. 


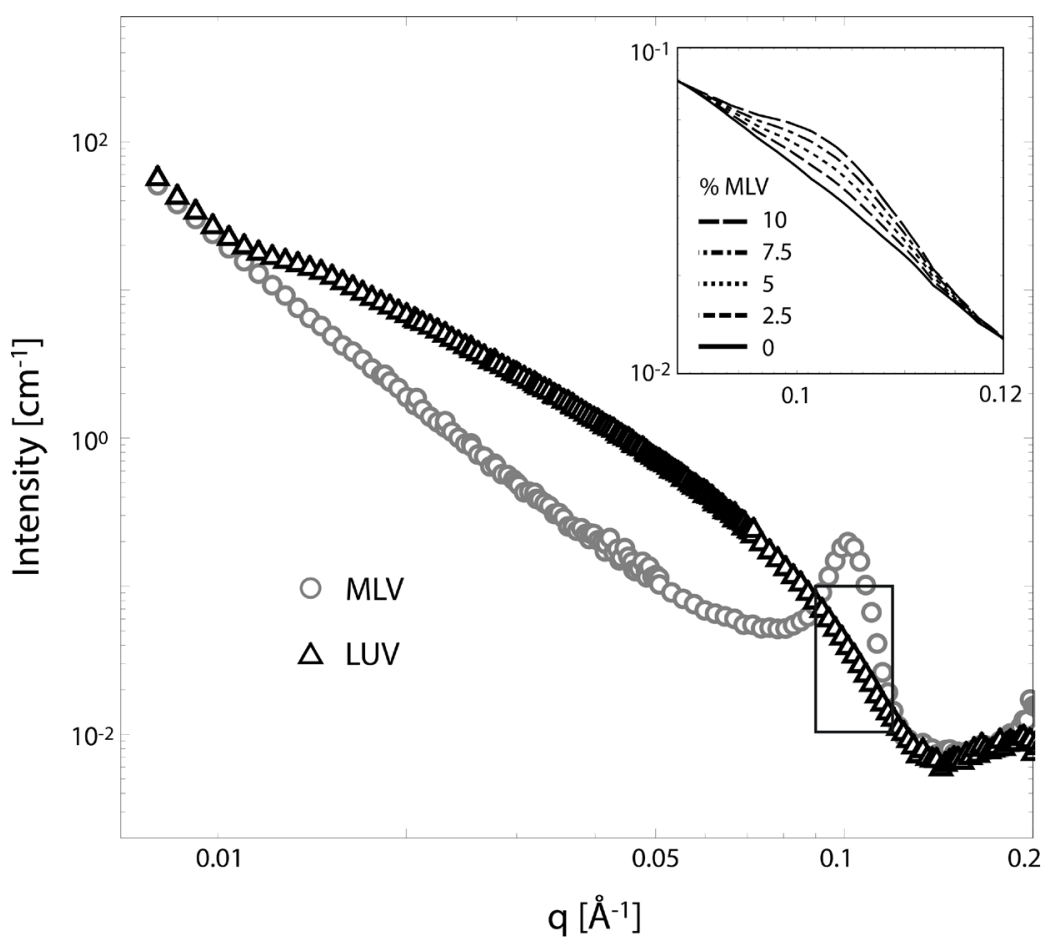

Figure S13 | SANS is sensitive to the presence of multilamellar vesicles (MLVs). Shown are SANS data for a $10 \mathrm{mM}$ POPC- $d C$ vesicle suspension in $\mathrm{D}_{2} \mathrm{O}$, before (MLVs, gray circles) and after (LUVs, black triangles) extrusion to produce $50 \mathrm{~nm}$ diameter LUVs. For MLVs, density correlations between the stacked bilayers give rise to Bragg scattering peaks at a length scale corresponding to integer multiples of the lamellar repeat distance (e.g., the first Bragg order at $q$ $\sim 0.1 \AA^{-1}$, corresponding to a lamellar repeat distance of $\sim 63 \AA$ ). Following extrusion to produce unilamellar vesicles, Bragg peaks are no longer observed, and vesicles exhibit the typical form factor for a dilute spherical shell particle. The inset shows a weighted sum of the black and gray curves as indicated in the inset legend, demonstrating the sensitivity of SANS to MLV contamination. 


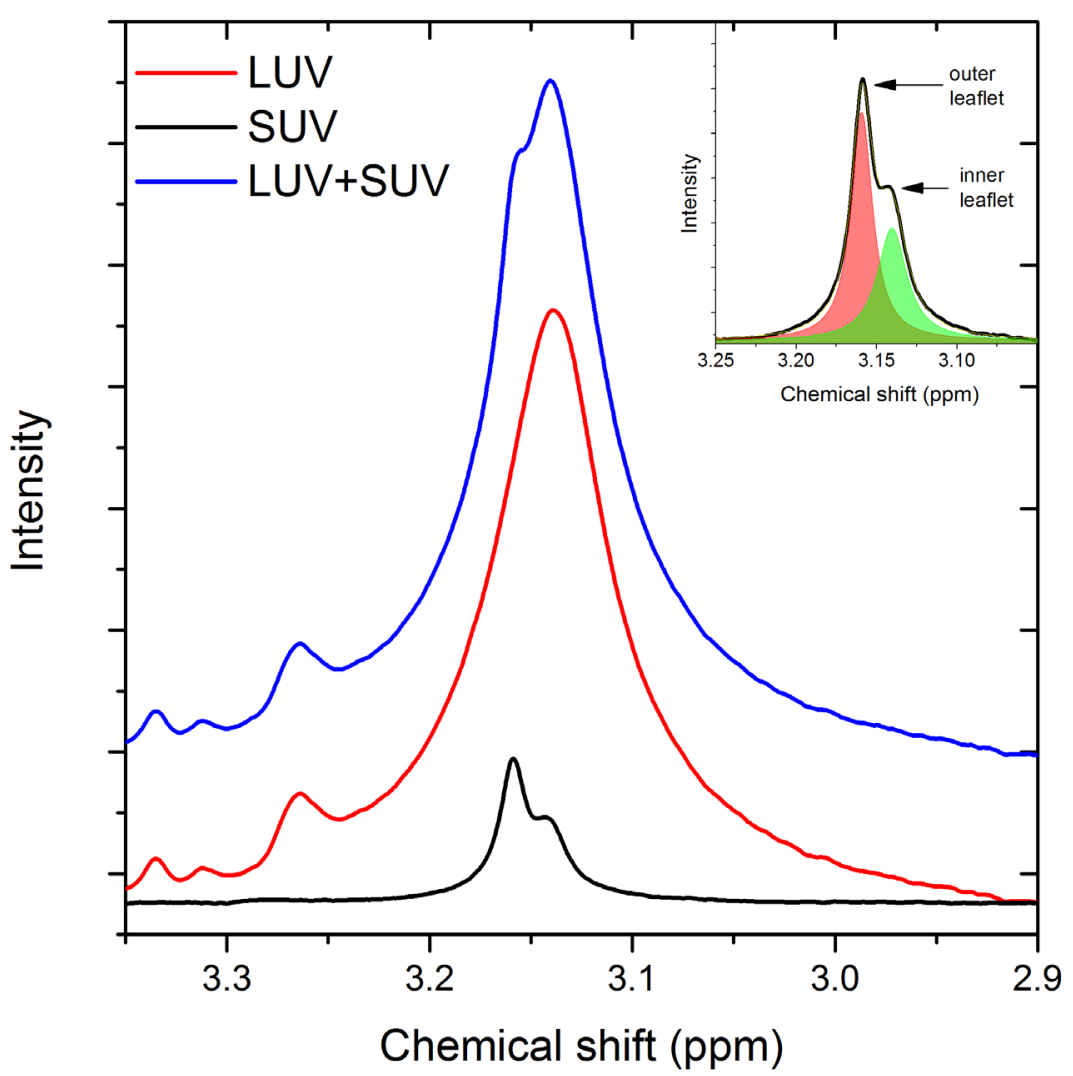

Figure S14 | ${ }^{1} \mathrm{H}-\mathrm{NMR}$ is sensitive to the presence of small unilamellar vesicles (SUVs). Shown are choline resonances from $14.6 \mathrm{mM}$ POPC LUVs (red) and $5.3 \mathrm{mM}$ POPC SUVs (black), as well as the sum of the LUV and SUV spectra (blue curve, offset for clarity). The shoulder at $3.16 \mathrm{ppm}$ demonstrates that $\sim 27 \mathrm{~mol} \%$ SUV can be easily identified in the presence of LUVs. Inset, small unilamellar vesicles (SUVs) give rise to a split choline resonance even in the absence of extravesicular shift reagent. This is due to both lipid number density and packing differences between the inner (green area) and outer (red area) leaflet. SUVs were obtained from an acceptor-free prep with omitted washing steps. 


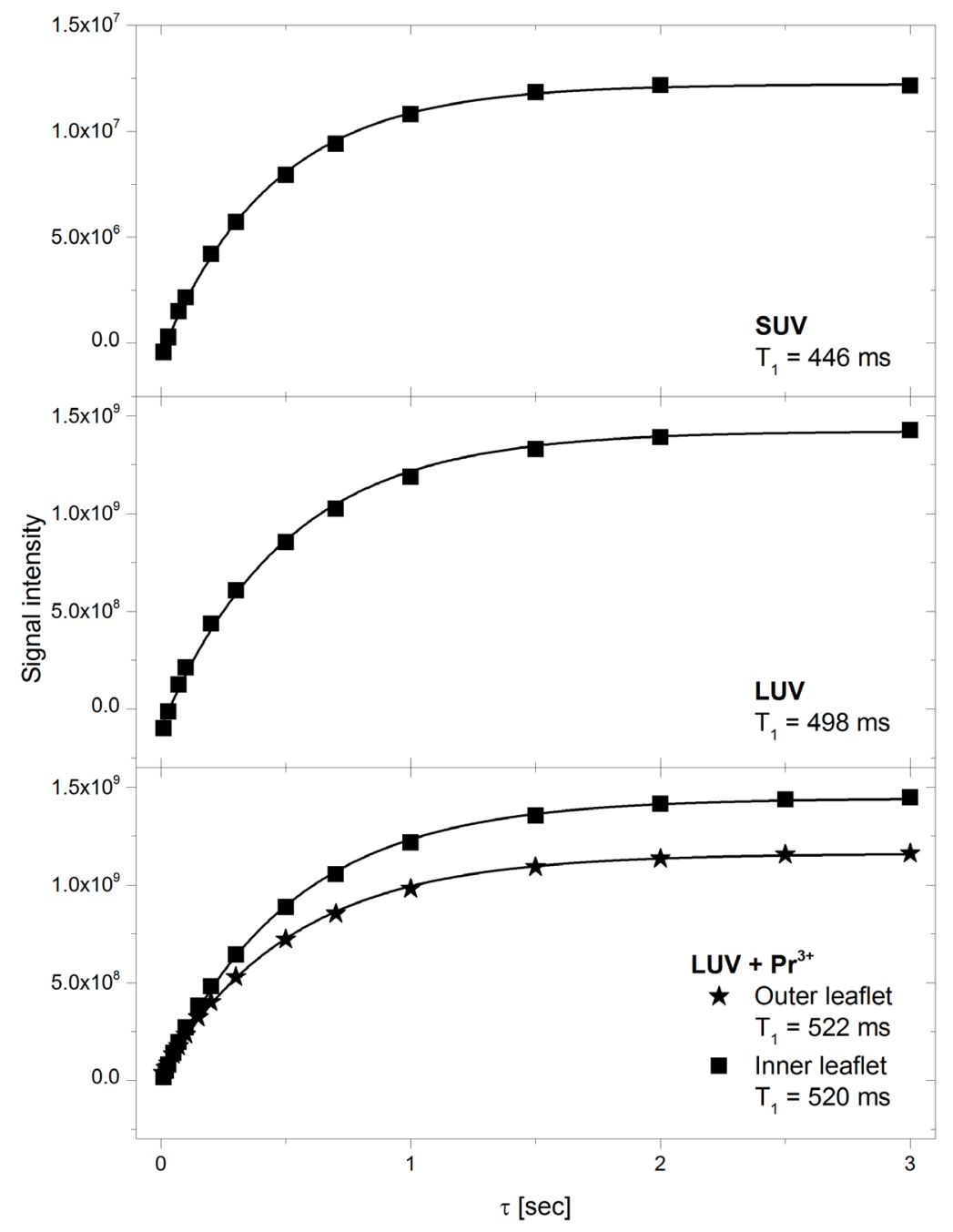

Figure S15 | ${ }^{1}$ H-NMR T1 relaxation measurements reveal SUVs and LUVs relax at the same rate, and $\mathrm{Pr}^{3+}$ does not change the $\mathrm{T} 1$ of the outer leaflet. Upper, SUVs generated from incubation of donor MLVs with $\mathrm{m} \beta \mathrm{CD}$. Middle, relaxation of symmetric LUVs. Bottom, relaxation of inner (square) and outer (star) leaflets of symmetric LUVs in the presence of extravesicular shift reagent $\mathrm{Pr}^{3+}$. Since the $\mathrm{T} 1$ relaxation times are identical, peak areas do not need to be scaled for the given spectrometer parameters. 


\section{S1. Methods}

Materials. 1-palmitoyl-2-oleoyl-sn-glycero-3-phosphocholine (16:0/18:1 PC, POPC), 1-palmitoyl-d31-2oleoyl-sn-glycero-3-phosphocholine [16:0(d31)/18:1 PC, POPC-dC], 1-palmitoyl-2-oleoyl-sn-glycero-3phosphocholine-1,1,2,2-d4-N,N,N-trimethyl-d9 [16:0/18:1 PC(d13), POPC-dH], 1-palmitoyl-d31-2oleoyl-sn-glycero-3-phosphocholine-1,1,2,2-d4-N,N,N-trimethyl-d9 [16:0(d31)/18:1 PC(d13), POPC$d H C$ ], 1,2-dipalmitoyl-d62-sn-glycero-3-phosphocholine [di-16:0(d31) PC, DPPC- $d C$ ], 1-palmitoyl-2oleoyl-sn-glycero-3-phospho-(1'-rac-glycerol) (sodium salt) [16:0/18:1 PG, POPG], and 1-palmitoyl-d312-oleoyl-sn-glycero-3-phospho-(1'-rac-glycerol) (sodium salt) [16:0(d31)/18:1 PG, POPG- $d C]$ were purchased from Avanti Polar Lipids (Alabaster, AL) and used as received. Lipid stock solutions were prepared by dissolving dry lipid powder in HPLC-grade chloroform, and phospholipid stock concentration was determined to within $1 \%$ by inorganic phosphate assay. ${ }^{3}$ Methyl-beta-cyclodextrin $(\mathrm{m} \beta \mathrm{CD})$ was purchased from Acros Organics (Thermo Fisher Scientific, Waltham, MA) and prepared as a $35 \mathrm{mM}$ stock solution in $\mathrm{H}_{2} \mathrm{O}$. Fig. $\mathrm{S} 1$ shows representative structures of lipids and $\mathrm{m} \beta C D$. Praseodymium(III) nitrate hexahydrate $\left\{\mathrm{Pr}\left(\mathrm{NO}_{3}\right)_{3} 6 \mathrm{H}_{2} \mathrm{O}\right\}\left(\mathrm{Pr}^{3+}\right)$ was purchased from Alfa Aesar (Ward Hill, MA) and prepared as a $100 \mathrm{mM}$ stock solution in $\mathrm{D}_{2} \mathrm{O}$. Centrifugal filter devices (Amicon Ultra-15, 100,000 Da molecular weight cutoff) were purchased from EMD Millipore (Billerica, MA) and washed seven times with $\mathrm{H}_{2} \mathrm{O}$ prior to use to remove trace glycerol. Ultrapure $\mathrm{H}_{2} \mathrm{O}$ was obtained from a High-Q purification system (Wilmette, IL), and $99.9 \% \mathrm{D}_{2} \mathrm{O}$ was purchased from Cambridge Isotopes (Andover, MA).

S2. Preparation of asymmetric liposomes. Phospholipid films were prepared by transferring the desired volumes of stock solutions to a glass scintillation vial with a syringe (Hamilton USA, Reno, NV) and then drying the organic solvent with an $\mathrm{N}_{2}$ stream and gentle heating, followed by overnight drying in vacuo ( $\sim 12$ h). Acceptor films were doped with $5 \mathrm{~mol} \%$ of the anionic lipid POPG to prevent formation of paucilamellar vesicles, which are known to form in extruded neutral liposomes of $100 \mathrm{~nm}$ diameter or larger. ${ }^{1,4}$ The isotopic variant of POPG used (i.e., POPG or POPG- $d C$ ) was always chosen to match that of the acceptor chains, and for data analysis its asymmetric distribution was assumed to be identical to that of the acceptor PC.

Donor multilamellar vesicles (MLVs) were prepared by hydrating a preheated $\left(50^{\circ} \mathrm{C}\right)$ lipid film with $20 \%(\mathrm{w} / \mathrm{w})$ sucrose solution to a lipid concentration of $20 \mathrm{mg} / \mathrm{mL}$, followed by vigorous vortexing to disperse the lipid. The resulting MLV suspension was incubated at $50{ }^{\circ} \mathrm{C}$ for $1 \mathrm{~h}$ with intermittent vortexing, and then subjected to three freeze/thaw cycles between -78 and $50{ }^{\circ} \mathrm{C}$ to facilitate equilibration of sucrose across lamellae. The MLV suspension was then diluted 20-fold with $\mathrm{H}_{2} \mathrm{O}$ and centrifuged at 
$20,000 \times \mathrm{g}$ for $30 \mathrm{~min}$ at $20^{\circ} \mathrm{C}$, after which time the supernatant was discarded, and the pellet was resuspended with $35 \mathrm{mM} \mathrm{m} \beta \mathrm{CD}$ solution to an 8:1 $\mathrm{m} \beta C D$ :lipid molar ratio. The MLV/m $\beta C D$ mixture was incubated at room temperature for $2 \mathrm{~h}$ with gentle stirring.

Acceptor large unilamellar vesicles (LUVs) were prepared by hydrating a preheated $\left(40^{\circ} \mathrm{C}\right)$ lipid film with $20 \mathrm{mM} \mathrm{NaCl}$ solution to a concentration of $10-12 \mathrm{mg} / \mathrm{mL}$, followed by vigorous vortexing to disperse the lipid. The resulting MLV suspension was incubated at $40{ }^{\circ} \mathrm{C}$ for $1 \mathrm{~h}$ with intermittent vortexing, and then subjected to five freeze/thaw cycles as described above. LUVs were prepared using a hand-held miniextruder (Avanti Polar Lipids, Alabaster, AL) assembled with a $100 \mathrm{~nm}$ pore-diameter polycarbonate filter, by passing the vesicle suspension through the filter 31 times at room temperature. LUV size was measured with dynamic light scattering (DLS) using a BI-200SM Research Goniometer and Laser Light Scattering system (Brookhaven Instruments, Holtsville, NY). Mean vesicle diameters were typically $\sim 125 \mathrm{~nm}$, with a relative polydispersity (distribution width divided by mean diameter) of $\sim$ 0.25 .

Asymmetric LUVs were prepared by mixing acceptor LUVs with the donor MLV/m $\beta C D$ suspension, at a donor:acceptor molar lipid ratio of 2:1 (for POPC donor/POPC acceptor samples) or 3:1 (for the DPPC donor/POPC acceptor sample). The mixture was stirred at room temperature for $1 \mathrm{~h}$, and then diluted 8-fold with $\mathrm{H}_{2} \mathrm{O}$ and centrifuged at 20,000 $\times \mathrm{g}$ for 30 min to pellet remaining donor MLVs. The pellet was discarded, and the supernatant (containing asymmetric LUVs, $\mathrm{m} \beta \mathrm{CD}$, and residual sucrose) was concentrated to $\sim 1 \mathrm{~mL}$ using a prewashed $100 \mathrm{~K}$ MWCO centrifugal filter device at 5,000 $\times$ g. Soluble contaminants (i.e., $\mathrm{m} \beta \mathrm{CD}$ and sucrose) were removed by successive dilution/concentration cycles, whereby the sample was diluted with $\mathrm{D}_{2} \mathrm{O}$ to the filter device's capacity $(\sim 11 \mathrm{~mL})$ and then centrifuged at $5,000 \times \mathrm{g}$ to obtain a final retentate volume of $0.5-1 \mathrm{~mL}$. The time for centrifugal filtration varied depending on the phase state of the lipids and the quantity of asymmetric vesicles being washed, and ranged from 30-60 min per wash. Typically, four such cycles reduced the $\mathrm{m} \beta \mathrm{CD}$ concentration by a factor of $>10$, and exchanged $>99 \%$ of $\mathrm{H}_{2} \mathrm{O}$ with $\mathrm{D}_{2} \mathrm{O}$. The asymmetric vesicle preparation is summarized in Fig. S2. The final yield was estimated as not less than half of the initial acceptor amount.

S3. Gas chromatography (GC). Phospholipids were converted to fatty acid methyl esters (FAMEs) via acid catalyzed methanolysis. Briefly, 5-10 $\mu \mathrm{L}$ of an aqueous vesicle suspension (containing 20-100 $\mu \mathrm{g}$ total lipid) was dispensed into a $13 \times 100 \mathrm{~mm}$ screw top glass culture tube, followed by addition of $1 \mathrm{~mL}$ methanolic $\mathrm{HCl}(1 \mathrm{M})$ prepared with concentrated $\mathrm{HCl}$ and methanol. ${ }^{5}$ The sample was vortexed, sealed under $\mathrm{Ar}$, and incubated at $85^{\circ} \mathrm{C}$ for $1 \mathrm{~h}$. After cooling to room temperature, $1 \mathrm{~mL} \mathrm{H}_{2} \mathrm{O}$ was added and 
the sample was vortexed. FAMEs were extracted with $1 \mathrm{~mL}$ hexane and vigorous vortexing, followed by low-speed centrifugation $(500 \times \mathrm{g})$ for $10 \mathrm{~min}$. Finally, $800 \mu \mathrm{L}$ of the upper (hexane) phase were transferred to an autosampler vial and brought to $1 \mathrm{~mL}$ with hexane, for injection into the $\mathrm{GC}$ column.

GC analysis was performed on an Agilent 5890A gas chromatograph (Santa Clara, CA) with a 5975C mass-sensitive detector operating in electron-impact mode. An HP-5MS capillary column (30 m $\times$ $0.25 \mathrm{~mm}, 0.25 \mu \mathrm{m}$ film thickness) was used with a helium carrier at $1 \mathrm{~mL} / \mathrm{min}$ and an inlet temperature of $270{ }^{\circ} \mathrm{C}$. A $1 \mu \mathrm{L}$ aliquot of FAME dissolved in hexane was injected in splitless mode using an Agilent 7693A automatic liquid sampler. After sample injection, the following column temperature program was initiated: $2 \mathrm{~min}$ at $60^{\circ} \mathrm{C} ; 20^{\circ} \mathrm{C} / \mathrm{min}$ to $170{ }^{\circ} \mathrm{C} ; 5^{\circ} \mathrm{C} / \mathrm{min}$ to $240{ }^{\circ} \mathrm{C} ; 30^{\circ} \mathrm{C} / \mathrm{min}$ to $300{ }^{\circ} \mathrm{C} ; 2 \mathrm{~min}$ at $300^{\circ} \mathrm{C}$, for a total run time of $25.5 \mathrm{~min}$. Total ion chromatogram peaks were assigned and integrated using GC/MSD ChemStation Enhanced Data Analysis software (Agilent Technologies, Santa Clara, CA).

S4. Determination of total bilayer composition using GC. Lipid mixture composition can be determined from GC, provided there are chemical or isotopic differences between chains of the constituent lipids. In principle, the mole fraction $\chi$ of each mixture component $i$ can be determined directly from a set of unique chain peak areas $\mathcal{A}=\left\{A_{i}\right\}$ :

$$
\chi_{i}=\frac{A_{i}}{\sum_{j} A_{j}},
$$

where $A_{i}$ denotes the $i^{\text {th }}$ chain peak area and the denominator is a sum over all mixture components $j$, $A_{j} \in \mathcal{A}$. For this relationship to be strictly valid, chain peak area fractions must vary linearly with mixture composition. In practice, we found a slight deviation from linearity which necessitated the use of standard curves (Fig. S3). Briefly, standard two-component FAME samples covering a range of compositions and containing 20-100 $\mu \mathrm{g}$ total lipid, were prepared by dispensing lipid stock solutions into glass culture tubes. Chloroform was removed by an $\mathrm{N}_{2}$ stream and gentle heating, followed by derivatization and extraction of FAMEs as described above. Peak area fractions $a_{i}$ were plotted $v s$. component mole fraction $\chi_{i}$ and fitted to a four-parameter function:

$$
a_{i}=\frac{a \chi_{i}^{2}+b \chi_{i}}{a \chi_{i}^{2}+b \chi_{i}+c\left(1-\chi_{i}\right)^{2}+d\left(1-\chi_{i}\right)}
$$


using Mathematica 10.0 (Wolfram Research, Champaign, IL) to obtain a standard curve, from which the composition of unknown samples was determined.

S5. Proton nuclear magnetic resonance spectroscopy $\left({ }^{1}\right.$ H-NMR). ${ }^{1} \mathrm{H}-\mathrm{NMR}$ spectra were collected on an Avance III $400 \mathrm{MHz}$ spectrometer (Bruker, Billerica, MA) using Bruker TopSpin acquisition software, and analyzed with TopSpin 3.2. Lipid suspensions in $\mathrm{D}_{2} \mathrm{O}$ were brought to a total volume of $600 \mu \mathrm{L}$ (for a total lipid concentration of $\sim 5 \mathrm{mM}$ ) and loaded into $5 \mathrm{~mm}$ NMR tubes (Wilmad LabGlass, Vineland, NJ). A standard ${ }^{1} \mathrm{H}$ pulse sequence with a $30^{\circ}$ flip angle and $1 \mathrm{~s}$ delay time was employed to collect $32-256$ transients at $21{ }^{\circ} \mathrm{C}$. ${ }^{1} \mathrm{H}-\mathrm{NMR}$ data were processed with a line-broadening parameter of $1 \mathrm{~Hz}$. The distribution of protiated choline between inner and outer vesicle leaflets (described further below) was determined by addition of the shift reagent $\operatorname{Pr}^{3+}{ }^{3}$ Briefly, $2 \mu \mathrm{L}$ of $100 \mathrm{mM} \operatorname{Pr}^{3+} / \mathrm{D}_{2} \mathrm{O}$ solution was dispensed directly into the NMR tube, which was then capped and inverted a minimum of three times to mix the contents. Typically, several such $\operatorname{Pr}^{3+}$ additions were made, with spectra obtained between titrations. Analysis of choline and $\mathrm{m} \beta \mathrm{CD}$ resonances was performed using Origin 8.5 software (OriginLab, Northampton, MA) and built-in nonlinear least-squares optimization tools. Resonances for the 9 identical choline protons were modeled using a single Lorentzian in the absence of $\operatorname{Pr}^{3+}$, or two Lorentzian peaks in the presence of $\operatorname{Pr}^{3}$. In most cases, spectra obtained from 2-3 successive $\operatorname{Pr}^{3+}$ titrations were separately modeled to determine the inner:outer leaflet ratio (and associated uncertainty) as described below. The reported outer leaflet fraction (Tables S2-S4) represents the average value obtained from these fits, with final uncertainty determined by error propagation.

S6. Evaluation of bilayer asymmetry with ${ }^{1} \mathbf{H}-\mathbf{N M R}$. The paramagnetic lanthanide ion $\operatorname{Pr}^{3+}$ interacts with choline protons, shifting their resonance downfield as shown in Figs. S4-S6. Because the ion's association and disassociation rates are fast relative to the NMR time scale, an average of shifted and unshifted resonances is obtained, and as such the extent of the observed shift depends on the molar ratio of $\operatorname{Pr}^{3+}$ and lipid. When $\operatorname{Pr}^{3+}$ is added externally to a vesicle suspension, the shift is selective for outer leaflet protiated choline, due to the low bilayer permeability of $\operatorname{Pr}^{3+}$ and the short-range distance dependence (inverse $r^{3}$ ) of the lanthanide-proton interaction. As a result, inner and outer leaflet protiated choline resonances can be separately resolved, ${ }^{7}$ and the integrated area $R$ of each resonance is proportional to the number of molecules having protiated headgroups in the corresponding leaflet. We define the outer leaflet peak fraction:

$$
f^{\text {out }}=\frac{R^{\text {out }}}{R^{\text {in }}+R^{\text {out }}}
$$


where the superscript denotes the inner (in) or outer (out) leaflet. If all bilayer components possess protiated headgroups, $f^{\text {out }}$ directly yields the mole fraction of all bilayer lipids found in the outer leaflet, $\mathrm{X}^{\text {out }}$ :

$$
\mathrm{X}^{\text {out }}=\frac{\sum_{j} N_{j}^{\text {out }}}{\sum_{j} N_{j}} \equiv f^{\text {out }},
$$

where $N$ and $N^{\text {out }}$ denote number of molecules in the whole bilayer and in the outer leaflet, respectively, and the summations are performed over all mixture components. (Note that this equation assumes that the chemical shifts of all cholines are identical.) For a bilayer with an equal number of lipids in both leaflets, $\mathrm{X}^{\text {out }}=0.5$. However, $\mathrm{X}^{\text {out }}$ can be influenced by geometric constraints (for example the differences in outer and inner leaflet volumes in highly curved vesicles ${ }^{8}$ ) or by differences in lipid packing in the inner and outer leaflets of asymmetric bilayers.

In a mixture of PC lipids, the assay is selective for a single species provided all other mixture components possess a deuterated choline. In this way, the distribution of donor and acceptor PC lipids can be separately established for differentially labeled samples. If one and only one mixture component (component $i$ ) possesses a protiated choline, we define the single-component outer leaflet peak fraction, $f_{i}^{\text {out }}$ as:

$$
f_{i}^{\text {out }}=\frac{N_{i}^{\text {out }}}{N_{i}}=\frac{\mathrm{X}^{\text {out }} \chi_{i}^{\text {out }}}{\chi_{i}}
$$

where all symbols are as defined above. Combining the two previous equations and rearranging gives an expression for the outer leaflet mole fraction of component $i, \chi_{i}^{\text {out }}$, in terms of experimentally determined quantities, $f_{i}^{\text {out }}$ and $f^{\text {out }}$ from NMR and $\chi_{i}$ from GC:

$$
\chi_{i}^{\text {out }}=\frac{f_{i}^{\text {out }} \chi_{i}}{f^{\text {out }}}
$$

S7. Small-angle neutron scattering (SANS). Neutron scattering experiments were performed at the BL6 extended Q-range small-angle neutron scattering (EQ-SANS) instrument of the Spallation Neutron Source (SNS) located at Oak Ridge National Laboratory (ORNL). Lipid vesicle samples were loaded into 
1 or $2 \mathrm{~mm}$ path length quartz banjo cells (Hellma USA, Plainview, NY) and mounted in a temperaturecontrolled cell holder with $\sim 1{ }^{\circ} \mathrm{C}$ accuracy. Data were taken at a $1.3 \mathrm{~m}$ sample-to-detector distance (SDD) with a 4.0-7.5 $\AA$ wavelength band, and at a 4.0 m SDD with a 10.0-13.5 $\AA$ wavelength, for a total scattering vector of $0.005<q<0.5 \AA^{-1}$. Scattered neutrons were collected with a two-dimensional $(1 \times 1$ m) ${ }^{3} \mathrm{He}$ position-sensitive detector (ORDELA, Inc., Oak Ridge, TN) with $256 \times 192$ pixels. The 2D data were reduced using software provided by ORNL. During reduction, data were corrected for detector pixel sensitivity, dark current, and sample transmission, and background scattering from water was subtracted. The one-dimensional scattered intensity $I$ vs. $q[q=4 \pi \sin (\theta) / \lambda$, where $\lambda$ is the neutron wavelength and $2 \theta$ is the scattering angle relative to the incident beam] was obtained by radial averaging of the corrected $2 \mathrm{D}$ data. The scattering contribution from residual $\mathrm{m} \beta \mathrm{CD}$ was accounted for by direct subtraction of a scaled $I(q)$ curve obtained for $\mathrm{m} \beta C D$ in aqueous solution.

S8. Determination of bilayer structure from SANS. In general, scattering from a dilute vesicle suspension contains contributions from both bilayer structure and vesicle size and shape. Provided the relevant length scales (i.e., the bilayer thickness and vesicle radius) are well-separated, the scattered intensity can be expressed as a product of form factors:

$$
I(q) \propto S(q)\left|F_{T S}(q)\right|^{2}\left|F_{F B}(q)\right|^{2}
$$

where $S(q)$ is an interparticle structure factor accounting for intervesicle interference (equal to unity in the infinite dilution limit), $F_{T S}$ is the scattering amplitude of a thin spherical shell, and $F_{F B}$ is the scattering amplitude of an infinite flat bilayer sheet. ${ }^{9} F_{T S}$ contains information about vesicle size and polydispersity, while $F_{F B}$ contains information about the distribution of matter within the bilayer. For the dilute, $100 \mathrm{~nm}$ diameter vesicles used in this study, $S(q)\left|F_{T S}(q)\right|^{2} \approx$ constant for $q>0.05 \AA^{-1}$, such that the contribution from $F_{F B}$ dominates the observed intensity at large scattering vectors. Therefore, a suitable expression for $F_{F B}(q)$ is needed to model the data at $q>0.05 \AA^{-1}$, in order to obtain information about bilayer asymmetry.

As a reciprocal space quantity, the flat bilayer form factor is related to the bilayer's real space matter distribution through a Fourier transform:

$$
\left|F_{F B}(q)\right|^{2}=\left(\int_{-D^{\text {in }}}^{D^{\text {out }}}\left[\rho(z)-\rho_{S}\right] \cos (q z) d z\right)^{2}+\left(\int_{-D^{\text {in }}}^{D^{\text {out }}}\left[\rho(z)-\rho_{S}\right] \sin (q z) d z\right)^{2}
$$


where $\rho(z)$ is the projected scattering length density (SLD) in the direction normal to the bilayer plane, $\rho_{S}$ is the scattering length density of the solvent (water), and the integral is evaluated over the full bilayer thickness. ${ }^{10}$ We simplify the asymmetric bilayer's SLD profile by considering four slabs of independent $\rho$ and thickness $D$, in addition to the SLD of the vesicle core and external solvent. This model is represented graphically in Fig. S9 and mathematically as:

$$
\rho(z)=\left\{\begin{array}{cc}
\rho_{\text {Core }} & z<-\left(D_{C}^{\text {in }}+D_{H}^{\text {in }}\right) \\
\rho_{H}^{\text {in }} & -\left(D_{C}^{\text {in }}+D_{H}^{\text {in }}\right) \leq z<-D_{C}^{\text {in }} \\
\rho_{C}^{\text {in }} & -D_{C}^{\text {in }} \leq z<0 \\
\rho_{C}^{\text {out }} & 0 \leq z<D_{C}^{\text {out }} \\
\rho_{H}^{\text {out }} & D_{C}^{\text {out }} \leq z<D_{C}^{\text {out }}+D_{H}^{\text {out }} \\
\rho_{S} & z \geq D_{C}^{\text {out }}+D_{H}^{\text {out }}
\end{array}\right.
$$

where superscripts in and out refer to the inner and outer leaflets, respectively. Provided the core SLD matches that of the solvent $\left(\rho_{\text {Core }}=\rho_{S}\right.$ ), the asymmetric form factor has an analytical solution:

$$
\begin{aligned}
\left|F_{F B}(q)\right|^{2}=\frac{1}{q^{2}} & {\left[\left\{\left(\rho_{C}^{\text {out }}-\rho_{C}^{\text {in }}\right)+\left(\rho_{C}^{\text {in }}-\rho_{H}^{\text {in }}\right) \cos \left(D_{C}^{\text {in }} q\right)-\left(\rho_{C}^{\text {out }}-\rho_{H}^{\text {out }}\right) \cos \left(D_{C}^{\text {out }} q\right)\right.\right.} \\
& \left.+\left(\rho_{H}^{\text {in }}-\rho_{S}\right) \cos \left(\left(D_{C}^{\text {in }}+D_{H}^{\text {in }}\right) q\right)-\left(\rho_{H}^{\text {out }}-\rho_{S}\right) \cos \left(\left(D_{C}^{\text {out }}+D_{H}^{\text {out }}\right) q\right)\right\}^{2} \\
& +\left\{\left(\rho_{C}^{\text {in }}-\rho_{H, \text { in }}\right) \sin \left(D_{C}^{\text {in }} q\right)+\left(\rho_{C}^{\text {out }}-\rho_{H}^{\text {out }}\right) \sin \left(D_{C}^{\text {out }} q\right)\right. \\
& \left.\left.+\left(\rho_{H}^{\text {in }}-\rho_{S}\right) \sin \left(\left(D_{C}^{\text {in }}+D_{H}^{\text {in }}\right) q\right)+\left(\rho_{H}^{\text {out }}-\rho_{S}\right) \sin \left(\left(D_{C}^{\text {out }}+D_{H}^{\text {out }}\right) q\right)\right\}^{2}\right] .
\end{aligned}
$$

We now seek expressions for the slab SLDs and thicknesses in terms of physically meaningful parameters related to the asymmetric bilayer's composition and structure. ${ }^{11}$ Assuming uniform lipid mixing with each leaflet, the volumes (or scattering lengths) of the outer and inner headgroup slabs are given as mole fraction-weighted sums of the component headgroup volumes (or scattering lengths) plus associated water:

$$
\begin{aligned}
& V_{H}^{j}=\sum_{i} \chi_{i}^{j} V_{H, i}+n_{W}^{j} V_{W}, \\
& b_{H}^{j}=\sum_{i} \chi_{i}^{j} b_{H, i}+n_{W}^{j} b_{W},
\end{aligned}
$$


where $V_{H}$ is the headgroup volume in $\AA^{3}, b$ is the coherent neutron scattering length in fm, $n_{W}$ is the average number of bound waters per headgroup, $\chi$ is the component mole fraction, the subscript $i$ indexes the bilayer's lipid components, and the superscript $j$ indexes the two leaflets. The analogous expressions for the hydrocarbon slabs are:

$$
\begin{aligned}
V_{C}^{j} & =\sum_{i} \chi_{i}^{j} V_{C, i}, \\
b_{C}^{j} & =\sum_{i} \chi_{i}^{j} b_{C, i} .
\end{aligned}
$$

The slab thicknesses and scattering length densities follow directly:

$$
\begin{gathered}
\rho_{H(C)}^{j}=b_{H(C)}^{j} / V_{H(C)}^{j}, \\
D_{H(C)}^{j}=V_{H(C)}^{j} / A_{L}^{j},
\end{gathered}
$$

where $D$ is the slab thickness in $\AA$, and $A_{L}$ is the average area per lipid in $\AA^{2}$. With lipid volumes and scattering lengths constrained by independent measurements (Table S1), the model has four free structural parameters $\left[A_{L}^{\text {in }}, A_{L}^{\text {out }}, n_{W}^{\text {in }}, n_{W}^{\text {out }}\right]$ in addition to the compositional parameters $\chi_{i}^{\text {in }}, \chi_{i}^{\text {out }}$. For a twocomponent bilayer (e.g., lipids A and B) in which the lipids are uniformly mixed, the compositional parameters are given by ( $c f$. Eqs. 4-6):

$$
\begin{gathered}
\chi_{A}^{\text {out }}=\frac{f_{A}^{\text {out }} \chi_{A}}{\mathrm{X}^{\text {out }}}, \\
\chi_{A}^{\text {in }}=\frac{\left(1-f_{A}^{\text {out }}\right) \chi_{A}}{\left(1-\mathrm{X}^{\text {out }}\right)}, \\
\chi_{B}^{\text {in out })}=1-\chi_{A}^{\text {in }(\text { out })},
\end{gathered}
$$

where $\chi_{A}$ and $f_{A}^{\text {out }}$ are obtained with GC and NMR experiments, respectively, and $\mathrm{X}^{\text {out }}(c f$. Eq. 4$)$ is given by the inner and outer leaflet areas per lipid:

$$
\mathrm{X}^{\text {out }}=\frac{1 / A_{L}^{\text {out }}}{1 / A_{L}^{\text {out }}+1 / A_{L}^{\text {in }}}
$$


Compositional parameters for isotopically asymmetric POPC bilayers obtained from GC, NMR, and SANS experiments are given in Tables S2-S3. Structural parameters obtained from SANS analysis of isotopically asymmetric POPC bilayers are given in Table S6, with data and best-fit curves shown in Fig. 3a. For comparison, structural parameters obtained from a joint refinement of symmetric POPC variants are found in Table S5, with data and best-fit curves shown in Fig. S10.

For $k$ coexisting bilayer phases, assuming negligible interference, the observed intensity is given by a weighted sum of asymmetric form factors for each phase:

$$
I_{o b s}(q) \propto\left|F_{o b s}(q)\right|^{2}=\sum_{k} \alpha_{k}\left|F_{F B, k}(q)\right|^{2}
$$

where $\alpha_{k}$ is the phase area fraction given. For a binary mixture of two coexisting phases (e.g., phases 1 and 2), four bilayer compartments must be considered, namely the outer and inner leaflets of phases 1 and 2. Using superscripts $i$ and $o$ to refer to the inner and outer leaflets, respectively, the unconstrained model has eight structural parameters $\left(A_{L}^{i 1}, A_{L}^{i 2}, A_{L}^{o 1}, A_{L}^{o 2}, n_{W}^{i 1}, n_{W}^{i 2}, n_{W}^{o 1}, n_{W}^{o 2}\right)$ and four compositional parameters $\left(\chi_{A}^{i 1}, \chi_{A}^{i 2}, \chi_{A}^{o 1}, \chi_{A}^{o 2}\right)$, in addition to the phase 1 area fraction $\alpha_{1}\left(\alpha_{2}=1-\alpha_{1}\right)$. Some parameters can be constrained by matter balance combined with independent measurements. For example, with the bilayer mole fraction and leaflet distribution of component A (i.e., $\chi_{A}$ and $f_{A}^{o}$ ) independently determined from GC and NMR experiments, $\alpha_{1}$ is given by:

$$
\alpha_{1}=\frac{A_{L}^{i 1} A_{L}^{o 1}\left[A_{L}^{i 2} \chi_{A}^{o 2}-\left(A_{L}^{i 2}+A_{L}^{o 2}\right) \chi_{A} f_{A}^{o}\right]}{A_{L}^{i 2} A_{L}^{o 1} A_{L}^{o 2} \chi_{A} f_{A}^{o}-A_{L}^{i 1}\left\{A_{L}^{o 1} A_{L}^{o 2} \chi_{A} f_{A}^{o}+A_{L}^{i 2}\left[A_{L}^{o 2} \chi_{A}^{o 1}-A_{L}^{o 1} \chi_{A}^{o 2}+\left(A_{L}^{o 1}-A_{L}^{o 2}\right) \chi_{A} f_{A}^{o}\right]\right\}}
$$

and the inner leaflet phase 2 mole fraction of component $\mathrm{A}$ is given by:

$=\frac{{\chi_{A}^{i 2}}_{L}^{i 2} A_{L}^{o 1}\left(\chi_{A}^{i 1}-\chi_{A}\right) \chi_{A}^{o 2}-A_{L}^{i 1} \chi_{A}\left[\left(A_{L}^{i 2}+A_{L}^{o 2}\right) \chi_{A}^{o 1}-A_{L}^{i 2} \chi_{A}^{o 2}\right]\left(f_{A}^{o}-1\right)-A_{L}^{o 1} \chi_{A}\left[\left(A_{L}^{i 2}+A_{L}^{o 2}\right) \chi_{A}^{i 1}-A_{L}^{i 2} \chi_{A}^{o 2}\right] f_{A}^{o}}{A_{L}^{i 1} A_{L}^{o 2} \chi_{A}^{o 1}-\left(A_{L}^{i 1}+A_{L}^{o 1}\right) A_{L}^{o 2} \chi_{A} f_{A}^{o}}$

leaving 11 free parameters, an unacceptably large number. Therefore, additional assumptions were made when modeling DPPC/POPC aLUVs in order to reduce the number of free parameters to a reasonable level: (1) the number of headgroup waters for both leaflets was fixed to 7; and (2) given the small amount of DPPC found in the inner leaflet by NMR, we assumed that the inner leaflet was a uniform fluid phase and that the DPPC-poor phase was symmetric, allowing us to jointly vary three parameters (i.e., $A_{L}^{i 1}=$ 
$\left.A_{L}^{i 2}=A_{L}^{o 1}\right)$. The final model had four adjustable parameters as indicated in Table $\mathrm{S} 7$, which lists all structural and compositional parameters obtained from the fit (data and best-fit curves are shown in Fig. 3b). Compositional parameters obtained from GC, NMR, and SANS analysis are given in Table S4, where $\mathrm{X}^{\text {out }}$ is given by:

$$
\mathrm{X}^{\text {out }}=\frac{\alpha_{1} / A_{L}^{o 1}+\left(1-\alpha_{1}\right) / A_{L}^{o 2}}{\alpha_{1}\left(1 / A_{L}^{o 1}+1 / A_{L}^{i 1}\right)+\left(1-\alpha_{1}\right)\left(1 / A_{L}^{o 2}+1 / A_{L}^{i 2}\right)}
$$

S9. Assessment of sample contamination. We define a contaminant as any impurity whose presence can affect (or hinder the determination of) the asymmetric LUV bilayer structure. In this context, the most problematic contaminants are $\mathrm{m} \beta \mathrm{CD}$ and residual donor or mixed donor/acceptor vesicles that resist sedimentation. These are typically lighter donor multilamellar vesicles (MLVs), or small unilamellar vesicles (SUVs, diameter $<30 \mathrm{~nm}$ ) formed during the exchange process. As a lipid carrier molecule, $\mathrm{m} \beta C D$ facilitates outer leaflet exchange and may perturb the bilayer structure, while residual vesicles can bias the determination of the asymmetric leaflet compositions.

We assessed $\mathrm{m} \beta C D$ contamination by establishing the ${ }^{1} \mathrm{H}-\mathrm{NMR}$ detection limits of specific $m \beta C D$ resonances in $\mathrm{D}_{2} \mathrm{O}$ in the absence and presence of LUVs (Fig. S12), from which we estimate a lower detection limit of 1:130 CD:lipid molar ratio. Asymmetric LUVs typically contained $<1: 10$ CD:lipid after three wash steps (Fig. S12 inset).

The presence of residual vesicles can be assessed with a variety of techniques. Vesicles containing multiple lamellae (including contaminating donor MLVs) exhibit a series of Bragg peaks in the SANS intensity at $q=2 \pi n / D$, where $D$ is the lamellar repeat distance and $n$ is an integer. As illustrated in Fig. S13, mixtures of LUVs and MLVs show characteristic excess scattering near the first Bragg order $\left(n=1, \sim 0.1 \AA^{-1}\right)$. The absence of Bragg scattering in asymmetric samples is confirmed by a good fit between data and model (which assumes unilamellar vesicles), as well as simple visual inspection of the data.

A less obvious form of residual vesicle contamination is the presence of SUVs with diameter < $30 \mathrm{~nm}$, which can be generated upon lengthy exposure of lipid vesicles to $\mathrm{m} \beta \mathrm{CD}$. GC measurements of donor-only control samples revealed $1-2 \%$ of the total donor mass in the recovered sample, which was subsequently identified as SUVs with ${ }^{1} \mathrm{H}-\mathrm{NMR}$ lineshape analysis (Fig. S14). Briefly, the SUV choline resonance is characterized by the appearance of two peaks even in the absence of shift reagent, 
attributable to packing differences in the inner and outer leaflets of highly curved vesicles. ${ }^{7}$ The SUV choline resonance width is $\sim 0.25 \mathrm{ppm}$ - considerably narrower than the LUV width - with inner and outer resonances separated by $0.019 \mathrm{ppm}(7.6 \mathrm{~Hz})$. However, despite their considerable line width differences, the similarity of the spin-lattice relaxation time (T1) values for LUV and SUVs (Fig. S15) precludes the ability to isolate the SUV component by varying the delay time. NMR measurements of asymmetric samples did not indicate the presence of SUVs (Fig. 2a, Figs. S4-S6), although low contamination levels may fall below the detection threshold (Fig. S14). If complete removal of SUVs is necessary, sucrose density gradients can be employed prior to $\mathrm{m} \beta \mathrm{CD}$ removal.

\section{References}

1 Kučerka, N., Nieh, M. P. \& Katsaras, J. Biochim. Biophys. Acta 1808, 2761-2771 (2011).

2 Tristram-Nagle, S., Liu, Y., Legleiter, J. \& Nagle, J.F. Biophys. J. 83, 3324-3335 (2002).

3 Kingsley, P. B. \& Feigenson, G. W. Chem. Phys. Lipids 24, 135-147 (1979).

4 Fogarty, J. C., Arjunwadkar, M., Pandit, S. A. \& Pan, J. Biochim. Biophys. Acta 1848, 662-672 (2015).

5 Ichihara, K. \& Fukubayashi, Y. J. Lipid Res. 51, 635-640 (2010).

$6 \quad$ Andrews, S.B., Faller, J.W., Gilliam, J.M. \& Barrnett, R.J. Proc. Natl. Acad. Sci. USA 70, 18141818 (1973).

7 Perly, B., Smith, I. C., Hughes, L., Burton, G. W. \& Ingold, K. U. Biochim. Biophys. Acta 819, 131-135 (1985).

8 Berden, J.A., Barker, R. W. \& Radda, G. K. Biochim. Biophys. Acta 375, 186-208 (1975).

9 Pencer, J., Krueger, S., Adams, C.P. \& Katsaras, J. J. Appl. Crystallogr. 39, 293-303 (2006).

10 Kučerka, N., Pencer, J., Sachs, J.N., Nagle, J.F. \& Katsaras, J. Langmuir 23, 1292-1299 (2007).

11 Kučerka, N., Nagle, J.F., Feller, S.E. \& Balgavý, P. Phys. Rev. E 69, 051903 (2004). 